\title{
Thermal Conductivity of Argillaceous Rocks: Determination Methodology Using In Situ Heating Tests
}

\author{
Benoit Garitte $\cdot$ Antonio Gens · Jean Vaunat • \\ Gilles Armand
}

Received: 12 November 2012 / Accepted: 12 November 2012/Published online: 9 December 2012

(C) Springer-Verlag Wien 2012

\begin{abstract}
This study focuses on the characterisation of thermal conductivity for three potential host rocks for radioactive waste disposal. First, the heat conduction process is reviewed on the basis of an analytical solution and key aspects related to anisotropic conduction are discussed. Then the existing information on the three rocks is summarised and a broad uncertainty range of thermal conductivity is estimated based on the mineralogical composition. Procedures to backanalyse the thermal conductivity on the basis of in situ heating tests are assessed and a methodology is put forward. Finally, this methodology is used to estimate the impact of experimental uncertainties and applied to the four in situ heating tests. In the three potential host rocks, a clear influence of the bedding planes was identified and anisotropic heat conduction was shown to be necessary to interpret the observed temperature field. Experimental uncertainties were also shown to induce a larger uncertainty on the anisotropy ratio than on the equivalent thermal conductivity defined as the geometric mean of the thermal conductivity in the three principal directions.
\end{abstract}

Keywords Callovo-Oxfordian Clay · Boom Clay · Opalinus Clay · Thermal conductivity ·

In situ experiments $\cdot$ Heat

B. Garitte $(\bowtie) \cdot$ A. Gens $\cdot$ J. Vaunat

Universidad Politecnica de Catalunya (UPC), Barcelona, Spain

e-mail: benoit.garitte@gmail.com

G. Armand

Agence nationale pour la gestion des déchets radioactifs (ANDRA), Bure, France

\section{Introduction}

Since the Second World War, mankind has produced radioactive waste. According to the International Atomic Energy Agency (IAEA 2009) radioactive waste is classified into different categories. High level waste (HLW) is defined as the waste with levels of activity concentration high enough to generate significant quantities of heat by the radioactive decay process or waste with large amounts of long lived radionuclides that need to be considered in the design of a disposal facility. Most of the HLW is produced by the defence industry and the power generation industry (IAEA 2007). Although most of the data for the defence sector is classified, a worldwide accumulation of $800,000 \mathrm{~m}^{3}$ of HLW since the early days is estimated. By the beginning of 2003, power generation is estimated to have produced 255,000 metric tons of heavy metal (MTHM) of spent fuel in total. In 2010, 441 reactors are in operation providing a power capacity of about $375 \mathrm{GWe}$ (Giga Watt electrical). A modern power reactor with a capacity of $1 \mathrm{GWe}$ generates approximately spent fuel assemblies containing around 30-50 MTHM per year. This results in an overall annual increase of waste of about 15,000 MTHM and in a total amount of spent fuel nowadays of about 400,000 MTHM. Current reprocessing procedures allow for the conversion of 100 MTHM spent fuel into $40 \mathrm{~m}^{3}$ of vitrified HLW. A total volume of approximately $960,000 \mathrm{~m}^{3}$ of HLW (that should be multiplied by some kind of security factor to account for the containment structure) will thus have to be accommodated in the different geological disposal facilities worldwide.

Generally, geological disposals will be organised as a grid of storage galleries. Considering the maximum temperature that the host rock is allowed to reach and the heat output per metre of gallery as constraining factors, the 
diameter of the galleries and the distance between galleries in the grid will be mostly dependent on the thermal conductivity of the host rock. As a consequence of the total volume of waste and of the economic impact of gallery diameter and their distance, the determination of the thermal conductivity of the considered host rocks and of its uncertainty is of crucial importance. In this work, an attempt to characterise the thermal conductivity of Callovo-Oxfordian Clay, Opalinus Clay and Boom Clay, potential host rocks for the French, Swiss and Belgian disposal facilities, on the basis of four in situ heating tests is presented. Although the proposed methodology is applied specifically to three argillaceous rocks, it may also be used for other rock types as long as conduction is the dominant heat transport mode and that convection and changes of thermal conductivity of the host rock may be neglected.

\section{Heat Transport in Argillaceous Rocks}

In general, two modes may appear as candidates for heat transport in porous media: convection and conduction. In
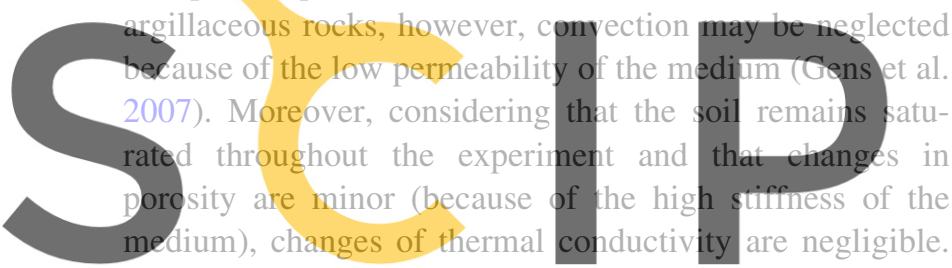

On the basis of these assumptions, the thermal conduction

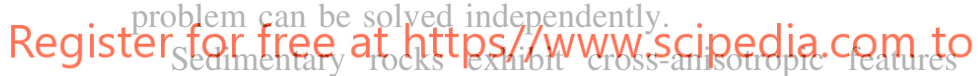

associated with the presence of bedding planes (Gens 2011)

including heat conduction properties. The analytical solution proposed by Booker and Savvidou (1985) for a point heat source in an infinite isotropic medium can be transformed (Carslaw and Jaeger 1946) to take anisotropy into account:

$\Delta T=\frac{q}{4 \cdot \pi \cdot \lambda_{0} \cdot R_{0}} \cdot \operatorname{erfc}\left(\frac{R_{0}}{2 \sqrt{\frac{\lambda_{0} \cdot t}{\rho \cdot C}}}\right)$

where $\Delta T$ is the temperature increment induced by $q$, the applied power (in $\mathrm{W}$ ). $\lambda_{0}$ is the equivalent thermal conductivity (in $\mathrm{W} / \mathrm{m} / \mathrm{K}$ ):

$\lambda_{0}=\sqrt[3]{\lambda_{\text {par }} \cdot \lambda_{\text {par }} \cdot \lambda_{\text {per }}}$

where $\lambda_{\text {par }}$ and $\lambda_{\text {per }}$ are the thermal conductivity in the bedding plane and in the perpendicular direction, respectively. $C$ is the heat capacity or specific heat (in $\mathrm{J} / \mathrm{kg} / \mathrm{K}$ ), $\rho$ is the density (in $\mathrm{kg} / \mathrm{m}^{3}$ ) and $t$ is the heating time (in s). erfc stands for complementary error function. The solution is calculated in a Cartesian reference system where $X$ - and $Y$-axes are considered in the bedding plane and $Z$ in the perpendicular direction. The transformed radius $R_{0}$ is a function of the isotropy-like distances $\xi, \eta$ and $\zeta$ in $x, y$ and $z$ direction, respectively:

$R_{0}=\sqrt{\xi^{2}+\eta^{2}+\zeta^{2}}$

$\xi, \eta$ and $\zeta$ are related to the real distances $x, y$ and $z$ through:

$x=\frac{\sqrt{\lambda_{\mathrm{par}}}}{\sqrt{\lambda_{0}}} \xi$
$y=\frac{\sqrt{\lambda_{\mathrm{par}}}}{\sqrt{\lambda_{0}}} \eta$

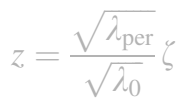

Two factors may be distinguished in Eq. (1): the complementary error function that expresses the transient behaviour and the pre-multiplying factor that gives the maximum temperature reached at steady state. Equation (1) is further analysed in Table 1 with emphasis on two

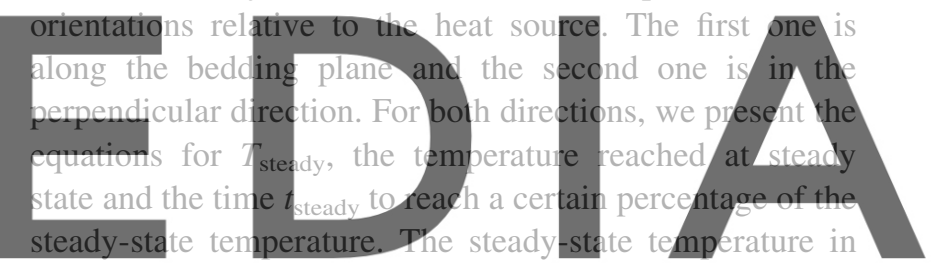
both directions is dependent on the geometric mean of the thermal condugtivity valdes in the transveral plane the the
download the version without the watermark
respective directions. The time to reach steady state is inversely proportional to the thermal conductivity (or thermal diffusivity) between the observation point and the heat source and proportional to the square of the distance between the observation point and the heat source.

More complex boundary value problems may be resolved using finite elements. Code_Bright (Olivella 1995 ) is a finite element code that allows solving partial differential equations in one, two or three dimensions. In this case, the relevant equation is the energy balance:

Table 1 Analysis of the temperature field in an anisotropic medium in the bedding plane and the perpendicular direction

\begin{tabular}{lll}
\hline & Parallel $\left(z=0, r=\sqrt{x^{2}+y^{2}}\right)$ & $\begin{array}{l}\text { Perpendicular } \\
(x \text { and } y=0)\end{array}$ \\
\hline$T_{\text {steady }}$ & $\frac{q}{4 \cdot \pi \cdot \sqrt{\lambda_{\text {par }} \cdot \lambda_{\text {per }} \cdot r}}$ & $\frac{q}{4 \cdot \pi \cdot \lambda_{\mathrm{par}} \cdot z}$ \\
$t_{\text {steady }}^{*}$ & $f \cdot \frac{r^{2} \cdot \rho \cdot C}{\lambda_{\text {par }}}$ & $f \cdot \frac{z^{2} \cdot \rho \cdot C}{\lambda_{\text {per }}}$ \\
\hline
\end{tabular}

$f=127.16$ to reach $95 \%$ of the steady-state temperature and $f=3,183.29$ to reach $99 \%$ of the steady-state temperature 
$\frac{\partial(\rho \cdot C \cdot T)}{\partial t}+\operatorname{div}\left(\overrightarrow{i_{\mathrm{c}}}\right)=f_{E}$

where $\rho$ is the density of the porous medium, $C$ the specific heat of the porous medium, $f_{E}$ a well/sink term of heat and $i_{\mathrm{c}}$ is the conductive flux of heat caused by temperature gradients:

$\overrightarrow{i_{\mathrm{c}}}=\left[\begin{array}{ccc}\lambda_{\mathrm{par}} & 0 & 0 \\ 0 & \lambda_{\mathrm{par}} & 0 \\ 0 & 0 & \lambda_{\text {per }}\end{array}\right] \cdot \nabla T$

where $\lambda_{\text {par }}$ and $\lambda_{\text {per }}$ are the thermal conductivity values in the bedding plane and in the perpendicular direction, respectively. Code_Bright was used in this work to model the different in situ heating tests.

\section{In Situ Heating Tests and Feedback from Laboratory}

Four in situ heating tests are analysed in this work:

- The ATLAS experiment in Boom Clay (De Bruyn and Labat 2002) performed in the HADES Underground Rock Laboratory (URL) in Belgium (Bernier et al.

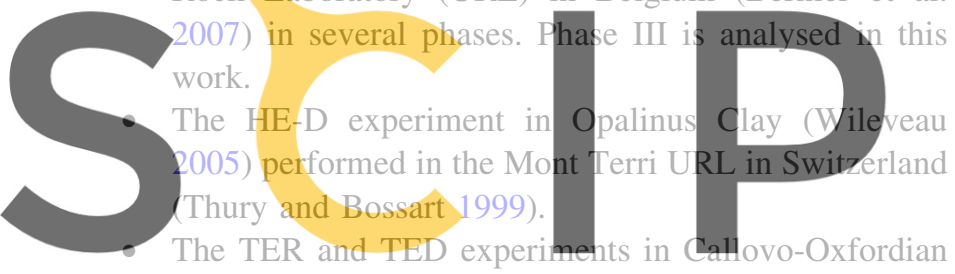
Clay (Conil et al. 2010) performed in the Meuse/Haute

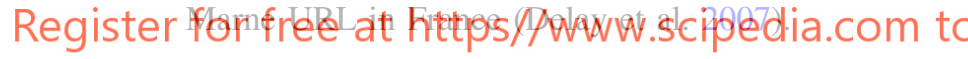

Callovo-Oxfordian Clay and Opalinus Clay overconsolidated Mesozoic argillaceous rock sedimented during the Middle Jurassic. Boom Clay is younger (Oligocene) and exhibits a lower overconsolidation ratio. An overview of the hydro-mechanical properties of the three materials may be found in Gens (2011). A general review of the three laboratories, THM processes and outstanding issues has been presented by Tsang et al. (2012). At the location of the URL, Boom Clay and Callovo-Oxfordian Clay did only experience weak tectonic activity and the bedding plane is thus subhorizontal. The Mont Terri URL instead is located in the Jura Mountain and the Opalinus Clay layer here underwent several folding events resulting in the Mont Terri anticline. In the HE-D area, values of the dip angle of typically $45^{\circ}$ were measured.

Each of the experiments considers the emplacement of a heating device in direct contact with the host rock in a horizontal borehole. In the TED experiment, three heaters were emplaced in parallel boreholes. The heating path of the tests is presented in Fig. 1a and b as a function of the time since heating started. The heating strategy of the

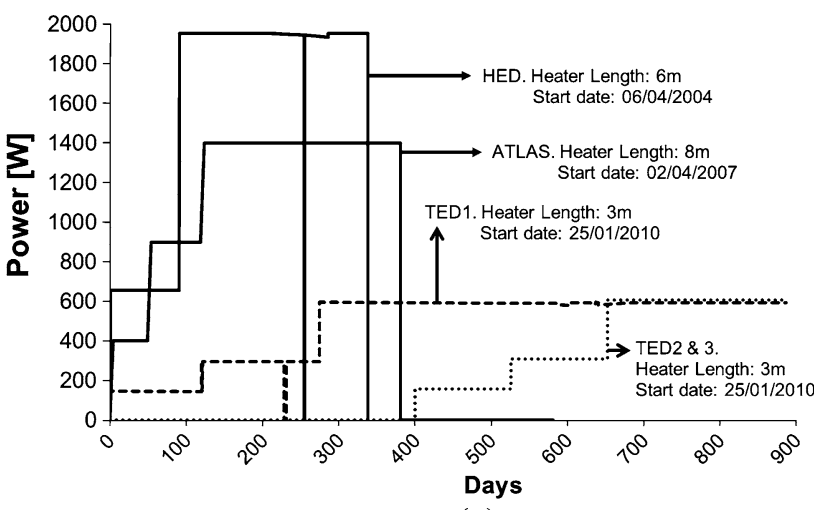

(a)

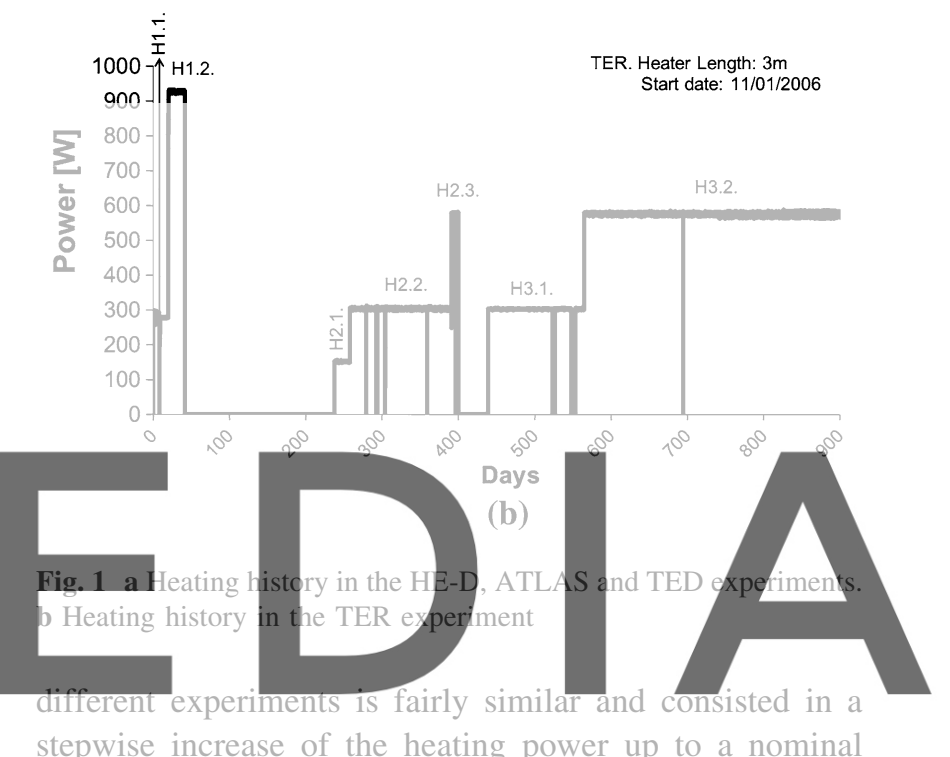

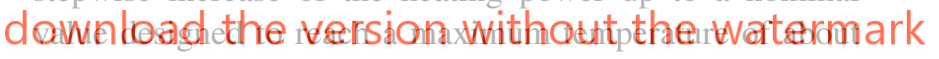

90-100 ${ }^{\circ} \mathrm{C}$ at heater-rock contact. The resulting nominal heating power related to the heating borehole wall surface was $293 \mathrm{~W} / \mathrm{m}^{2}$ in the ATLAS experiment, $345 \mathrm{~W} / \mathrm{m}^{2}$ in the HE-D experiment and $436 \mathrm{~W} / \mathrm{m}^{2}$ in the TER and TED experiments. As a consequence of the long duration of heating, unexpected shutdown events occurred in most of the experiments. The nomenclature of the different heating phases of the TER experiment (H1.1., H1.2., H2.1., H2.2., H2.3., H3.1. and H3.2.) has been added in Fig. 1b as this experiment is used to illustrate the determination methodology of the thermal conductivity. That methodology relies on the temperature measurements carried out in the rock mass around the heating boreholes. The number of emplaced temperature sensors in each experiment is given in Table 2.

Table 2 Number of temperature sensors in the rock mass in the different in situ experiments

\begin{tabular}{llll}
\hline ATLAS & TER & TED & HE-D \\
\hline 24 & 20 & 108 & 26 \\
\hline
\end{tabular}


Two parameters are directly related to the thermal conduction problem: specific heat and thermal conductivity. Both parameters are dependent on the composition of the porous materials: the porosity itself, the mineralogy of the solid phase and the fluids present in the pores. Specific heat is the amount of heat required to raise the temperature of the unit mass of the material by a given amount (usually one degree). As a consequence of the storage nature of this parameter, a volumetric average considering the different constituents may be applied:

$$
\begin{aligned}
\rho \cdot C= & \rho_{\mathrm{s}} \cdot(1-\phi) \cdot C_{\mathrm{s}}+\rho_{\mathrm{w}} \cdot \phi \cdot S_{r} \cdot C_{\mathrm{w}}+\rho_{\mathrm{a}} \cdot \phi \\
& \cdot\left(1-S_{r}\right) \cdot C_{\mathrm{a}}
\end{aligned}
$$

where the "s", "w" and "a" subscripts stand for solid, water and air, respectively. $\varphi$ is the porosity and $S_{r}$ is the degree of saturation. Thermal conductivity is the property of a material that indicates its ability to conduct heat. In this case, it is clear that there is an influence of the material composition, but also of the arrangement of the different constituents. Farouki (1986) proposed a formula for the isotropic thermal conductivity that can be applied to a random mixture of several materials. Applied to a porous medium, it states:

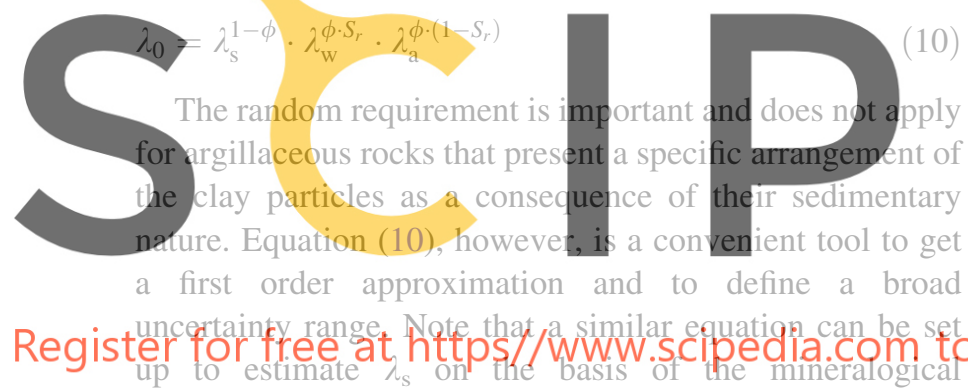
composition. Significant variations of carbonates or quartz which are relatively high conductive minerais are known to occur in argillaceous rock as a function of the sediment level and should be taken into account. Average values of the mineralogical composition have been indicated for the three rocks in Table 3. Whenever possible, the data are taken from samples at the location of the in situ heating experiments. Considering the

Table 3 Indicative mineralogy (\% weight of total solid) of the three argillaceous rocks in terms of the three main components and their porosity

\begin{tabular}{lcll}
\hline & Boom Clay & Opalinus Clay & Callovo-Oxfordian Clay \\
\hline Clay & 70 & $67.5(40-80)$ & $55(30-60)$ \\
Calcite & 0 & $14(5-40)$ & $28(20-75)$ \\
Quartz & 30 & $18.5(10-40)$ & $17(7-35)$ \\
Porosity & 40 & 15 & 15
\end{tabular}

Variation range is indicated for Opalinus Clay and Callovo-Oxfordian Clay. Data are collected from Romero (1999), Wileveau (2005) and ANDRA (2009) mineralogy and accounting for a thermal conductivity of the clay component of $1.1 \mathrm{~W} / \mathrm{m} / \mathrm{K}$, of $0.7 \mathrm{~W} / \mathrm{m} / \mathrm{K}$ for the water and of 3.75 and $8 \mathrm{~W} / \mathrm{m} / \mathrm{K}$ for calcite and quartz crystals (Robertson 1988), a first order approximation of the saturated thermal conductivity of the three rocks can be calculated. On the basis of this estimation, CallovoOxfordian Clay and Opalinus Clay exhibit larger conductivity values of 1.8 and $1.6 \mathrm{~W} / \mathrm{m} / \mathrm{K}$, respectively. Boom Clay has a slightly lower thermal conductivity: 1.3 $\mathrm{W} / \mathrm{m} / \mathrm{K}$ as a consequence mainly of its larger water content.

Different laboratory experiment campaigns have been carried out on saturated Callovo-Oxfordian Clay samples. They are summarised in Table 4. A clear influence of the heat flow orientation with respect to bedding was observed. The laboratory experiments realised on Opalinus Clay samples are summarised in Jobmann and Polster (2007) and Bock (2009). Average values of 2.15 and $1.2 \mathrm{~W} / \mathrm{m} / \mathrm{K}$ are indicated for the parallel and the perpendicular thermal conductivity values of Opalinus Clay, respectively. In Boom Clay, there are a limited number of laboratory experiments set up to determine the thermal conductivity. Lima Amorim (2011) and Van Cauteren (1994) measured

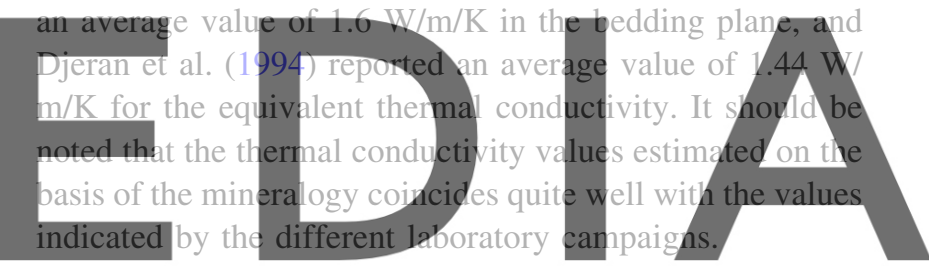

The different experimentalists mentioned above concurred in defecting a very. small temperature dependency of download the versiog without the watermark

standard deviation measured in the different laboratory campaigns. Moreover, in all cases, the specific heat of the solid phase of the rocks was close to $800-820 \mathrm{~J} / \mathrm{kg} / \mathrm{K}$, resulting in values for the saturated porous materials of about 1,000 J/kg/K for the Opalinus Clay (Fernandez 2011;

Table 4 Laboratory measurements of thermal conductivity in Callovo-Oxfordian Clay in the TED area (Conil et al. 2010) and in the

\begin{tabular}{|c|c|c|c|c|c|c|}
\hline & $\lambda_{\mathrm{par}}$ & $\lambda_{\text {per }}$ & $\lambda_{0}$ & $\lambda_{h /} \lambda_{v}$ & $n_{\text {par }}$ & $n_{\text {per }}$ \\
\hline \multicolumn{7}{|c|}{ TED area (DBE) } \\
\hline Average & 1.96 & 1.28 & 1.69 & 1.52 & 14 & 12 \\
\hline SD & 0.08 & 0.07 & 0.06 & 0.08 & & \\
\hline \multicolumn{7}{|c|}{ TED area (LAEGO) } \\
\hline Average & 1.89 & 1.26 & & & 5 & 3 \\
\hline SD & 0.05 & 0.04 & & & & \\
\hline \multicolumn{7}{|c|}{ TER area (LAEGO) } \\
\hline Average & 1.91 & 1.25 & & & 4 & 3 \\
\hline
\end{tabular}
TER area (Auvray et al. 2005)

$n_{\text {par }}$ and $n_{\text {per }}$ are the number of measurements parallel and perpendicular to the bedding, respectively 
Bock 2009) and the Callovo-Oxfordian Clay (Conil et al. 2010; Auvray et al. 2005) and 1,450 J/kg/K for Boom Clay (Van Cauteren 1994). Because of the lower uncertainty on the specific heat that depends exclusively on the medium composition and not on the grain arrangement, these values were assumed to be satisfactory estimates.

\section{Determination Methodology}

\subsection{Shortcomings of a Global Analysis}

The first attempt to determine the thermal conductivity was done on the basis of the temperature measurements in the TER in situ heating experiment. A series of thermal 3D finite element computations were run, solving the diffusion Eq. (7) and accounting for anisotropic heat conduction (8). In each of the computations, the thermal conductivity values (in the direction parallel and perpendicular to the bedding) were changed covering a wide range well beyond the thermal conductivity uncertainty. The parallel thermal conductivity was varied between 0.6 and $4 \mathrm{~W} / \mathrm{m} / \mathrm{K}$ and the perpendicular thermal conductivity between 0.5 and $3 \mathrm{~W} / \mathrm{m} / \mathrm{K}$. Nine hun-

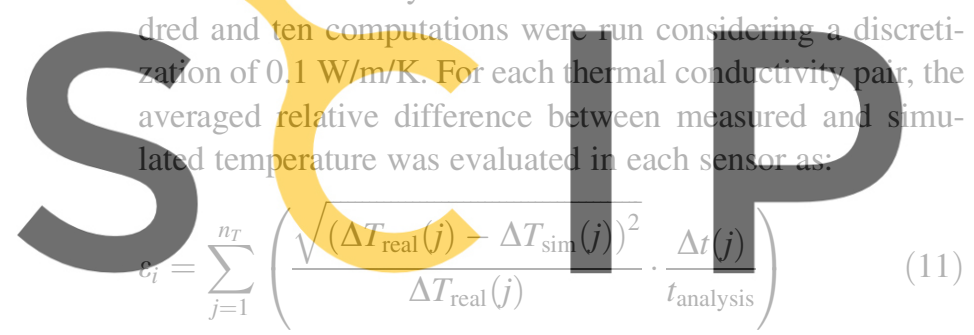

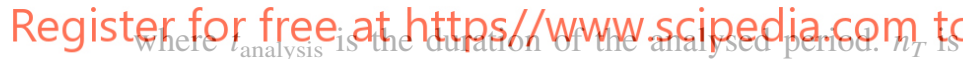
the number of measurements in the considered sensor during the analysed period. $\Delta T_{\text {reai }}(j)$ is the measured temperature increment since the start of the analysed period at time $t_{j}$ and $\Delta T_{\text {sim }}(j)$ is the simulated temperature increment at $t_{j} \cdot \frac{\Delta t(j)}{t_{\text {analysis }}}$ is a time factor used to weight measurement $j$, where $\Delta t(j)$ is computed as:

$\Delta t(j)=\frac{t_{j+1}-t_{j-1}}{2}$

This time weighting factor is introduced to avoid biasing the calculations in favour of the periods in which more frequent measurements were taken. The temperature differences can be averaged over several sensors:

$\varepsilon=\frac{\sum_{i=1}^{n_{\mathrm{MP}}} \varepsilon_{i}}{n_{\mathrm{MP}}}$

where $n_{\mathrm{MP}}$ is the number of sensors considered.

Average relative difference maps were built for each sensor of the TER experiment by applying Eq. (11) to each of the 910 simulations. The analysed period covers all heating phases H1.1., H1.2., H2.1., H2.2., H2.3. and H3.1.
A best-fitting thermal conductivity pair of values could only be determined for 10 of the 20 sensors. The difference maps for these sensors are presented in Fig. 2. For other sensors, the minimum in the difference maps was located outside the thermal conductivity domain considered. Malfunctioning of the sensor or an error in the sensor location was put forward as possible explanation. The dispersion of the determined thermal conductivity values is thus significant.

Even in the sensors indicating a minimum inside the investigated thermal conductivity domain, a significant scatter is observed. The best-fitting thermal conductivity pairs determined from the average relative difference maps are summarised in Table 5. Sensors located in the same bedding plane as the heater seem to indicate a parallel thermal conductivity value between 1.8 and $2.1 \mathrm{~W} / \mathrm{m} / \mathrm{K}$. Perpendicular sensors in turn indicate a somewhat higher parallel thermal conductivity: $2.6-2.7 \mathrm{~W} / \mathrm{m} / \mathrm{K}$. The perpendicular thermal conductivity varies between 0.5 and 1.4 $\mathrm{W} / \mathrm{m} / \mathrm{K}$ and it does not exhibit any particular pattern related to the sensor location relative to the heater.

Although the different sensors showed a very uneven behaviour, Eq. (13) was used to build a difference map

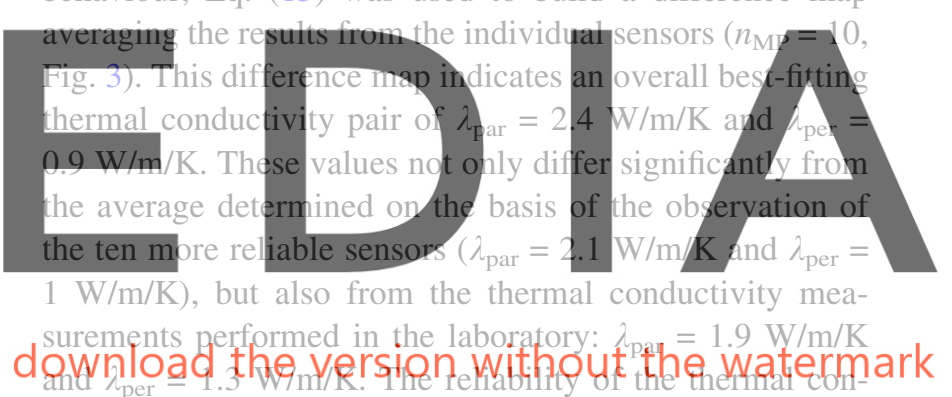

ductivity determination from the overall difference mini-

mum may thus be questioned. Moreover, the determination of the minimum is not very precise, in particular along the direction indicated with a dotted line. Cross sections of the difference map along the two axes of the minimum valley have been plotted in Fig. 4 to illustrate the difference in dispersion along the axes. The flatness of the valley bottom along the dotted line does not allow determining the thermal conductivity with a precision lower than $0.5 \mathrm{~W} / \mathrm{m} / \mathrm{K}$.

Each heating phase was also investigated separately in each sensor. Some sensors (e.g. TER1201TEM2 in Fig. 5) show a very consistent behaviour along the different heating phases. The best-fitting thermal conductivity pair in each heating phase is quite similar to the one determined for all the heating phases together. It is noteworthy that short heating phases (H1.1. and H2.1._-20 days each) seem to provide a precise determination of the perpendicular thermal conductivity and that longer heating phases (H2.2. and H3.1.) indicate a combination of parallel and perpendicular thermal conductivity pairs. Other sensors (e.g. 
Fig. 2 Map of average relative difference in each sensor considering the entire heating history in TER
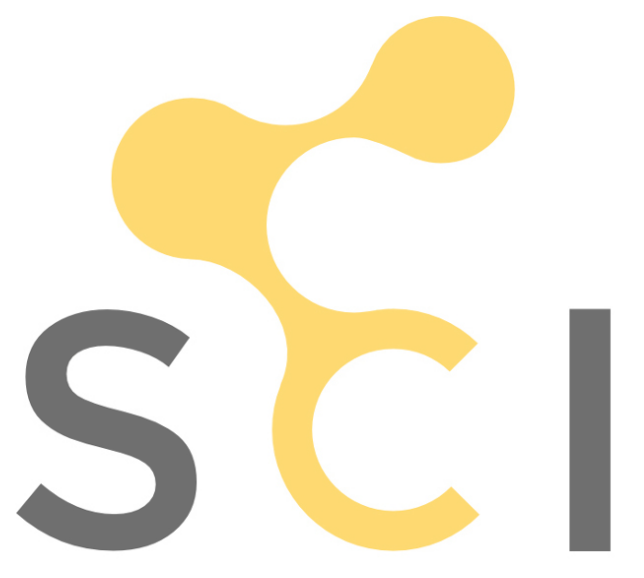

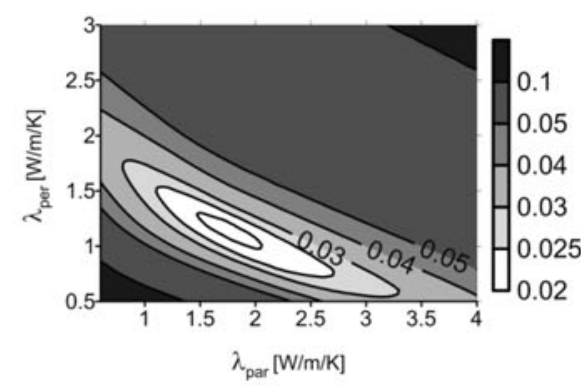

TER1201TEM2

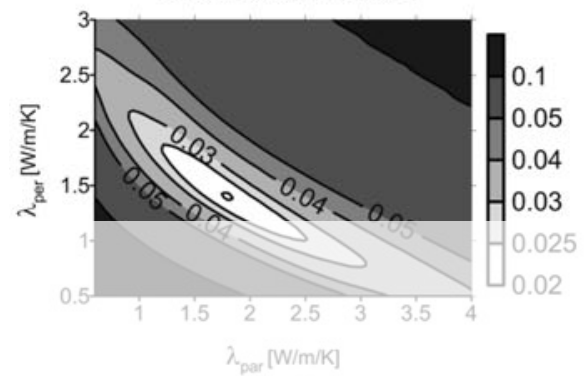

TER1201TEM3

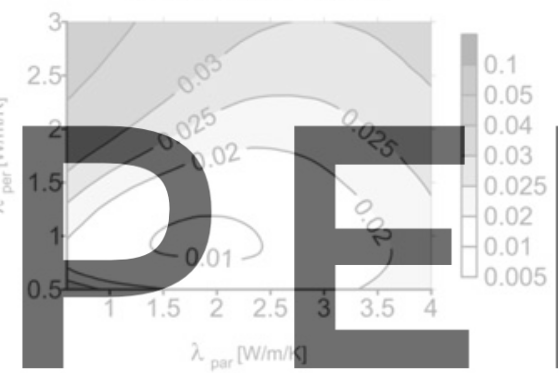

TER1201TEM5

TER1203TEM3

Register for free at https//www.scipedia.com to download the version without the watermark

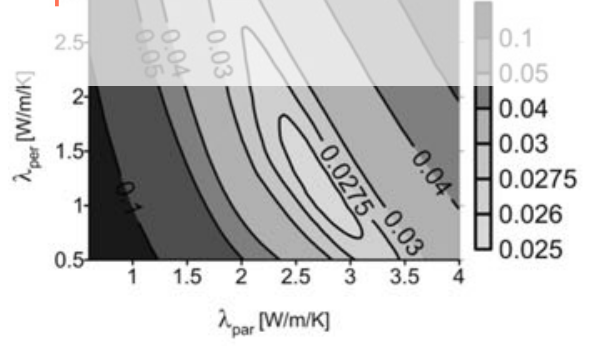

TER1203TEM4

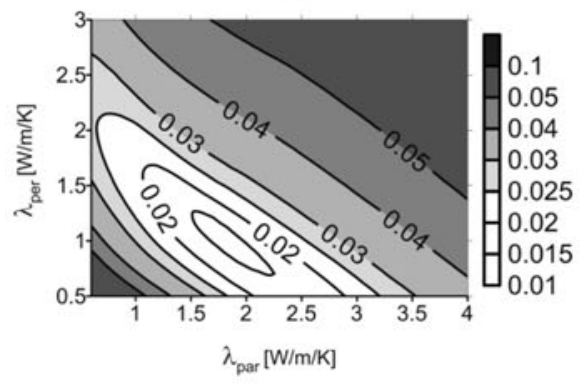

TER1404TEM1

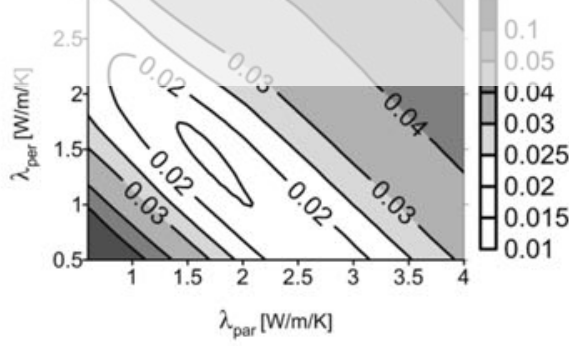

TER1403TEM1

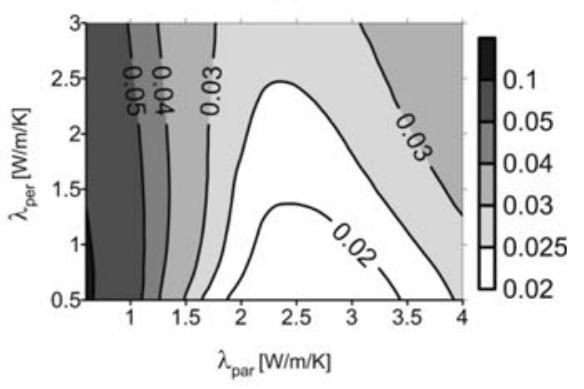

TER1405TEM1 
Table 5 Best-fitting thermal conductivity pair in each sensor on the basis of Fig. 2

\begin{tabular}{|c|c|c|c|c|c|}
\hline & $\lambda_{\text {par }}(\mathrm{W} / \mathrm{m} / \mathrm{K})$ & $\lambda_{\text {per }}(\mathrm{W} / \mathrm{m} / \mathrm{K})$ & $\begin{array}{l}\text { Sensor orientation } \\
\text { with respect } \\
\text { to bedding }\end{array}$ & $\begin{array}{l}\text { Radial distance } \\
\text { to heater }(\mathrm{m})\end{array}$ & $\begin{array}{l}\text { Distance to transversal } \\
\text { plane through } \\
\text { the centre } \\
\text { of the heater }(\mathrm{m})\end{array}$ \\
\hline TER1201TEM01 & 1.8 & 0.8 & Parallel & 0.49 & -3.01 \\
\hline TER1201TEM02 & 1.8 & 1.2 & Parallel & 0.53 & -1.51 \\
\hline TER1201TEM03 & 1.8 & 1.4 & Parallel & 0.57 & -0.01 \\
\hline TER1201TEM04 & 2.1 & 0.9 & Parallel & 0.61 & 1.49 \\
\hline TER1201TEM05 & 1.9 & 1 & Parallel & 0.64 & 2.99 \\
\hline TER1203TEM03 & 2.7 & 0.7 & Perpendicular & 0.57 & -0.10 \\
\hline TER1203TEM04 & 2.6 & 1.2 & Perpendicular & 0.63 & 1.40 \\
\hline TER1403TEM01 & 1.7 & 1.4 & $45^{\circ}$ & 1.31 & -1.39 \\
\hline TER1404TEM01 & 1.9 & 1 & Perpendicular & 0.66 & -2.09 \\
\hline TER1405TEM01 & 2.7 & 0.5 & Perpendicular & 1.41 & -1.19 \\
\hline Average & 2.1 & 1 & & & \\
\hline Min & 1.7 & 0.5 & & & \\
\hline Max & 2.7 & 1.4 & & & \\
\hline
\end{tabular}

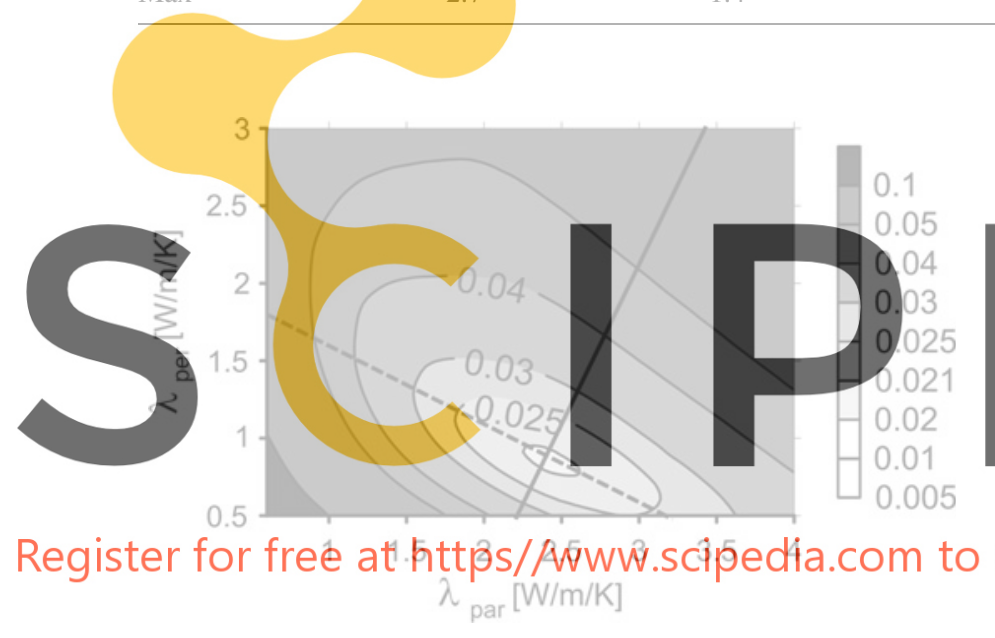

Fig. 3 Map of average relative difference considering all sensors and the entire heating history in TER

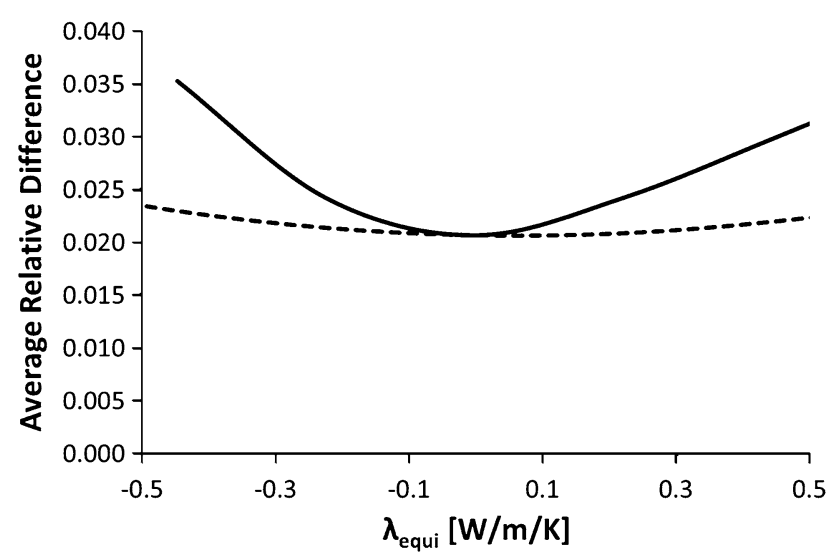

Fig. 4 Cross sections of the difference map from Fig. 3 along the dotted and full profile lines. $\lambda_{\text {equi }}$ is taken 0 at the cross over and $\lambda_{\text {equi }}= \pm \sqrt{\lambda_{\text {par }}^{2}+\lambda_{\text {per }}^{2}}$
TER1203TEM4 in Fig. 6) exhibit a significantly erratic behaviour depending on the heating phase used for the

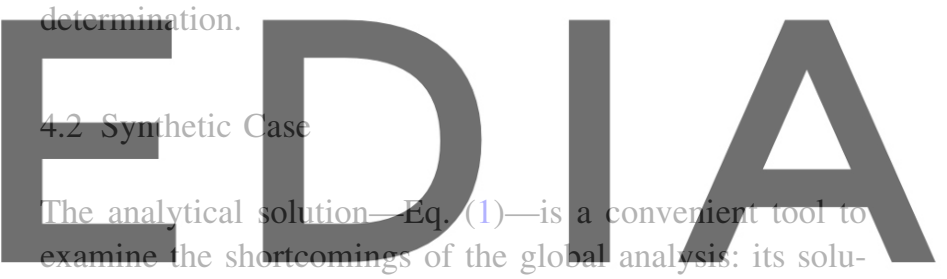

tion is fast and no numerical errors are introduced. First, a

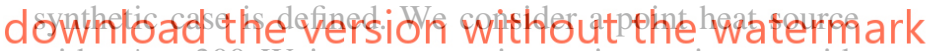
with $q^{*}=300 \mathrm{~W}$ in a cross-anisotropic continuum with $\lambda_{\text {par }}^{*}=2.1 \mathrm{~W} / \mathrm{m} / \mathrm{K}, \lambda_{\text {per }}^{*}=1 \mathrm{~W} / \mathrm{m} / \mathrm{K}$ and $C^{*}=1,000 \mathrm{~J} / \mathrm{kg} / \mathrm{K}$.

The temperature evolution $T^{*}$ has been computed with the analytical solution at $x^{*}=0.5 \mathrm{~m}$ and $x^{*}=2 \mathrm{~m}\left(y^{*}=z^{*}=0\right.$ $\mathrm{m})$ in the bedding plane and at $z^{*}=0.5 \mathrm{~m}$ and $z^{*}=2 \mathrm{~m}\left(y^{*}=\right.$ $x^{*}=0 \mathrm{~m}$ ) in the perpendicular direction (Fig. 7). $T^{*}$ has been compared with results from a finite element computation to discard code-related errors (Fig. 7, full lines). The superscript $*$ indicates values used to define the synthetic case, and when a position is indicated and no information is given about a specific coordinate, it should be considered 0 .

In a second step, the measured temperature increment $\Delta T^{*}$ is compared with the temperature increment $\Delta T$ calculated with the analytical solution using different thermal conductivity values. The comparison is carried out at a particular time $(t)$ using the following expression:

$\varepsilon(t)=\left.\frac{\sqrt{(\Delta T *-\Delta T)^{2}}}{\Delta T *}\right|_{t}$ 
Fig. 5 Map of average relative difference in sensor TER1201TEM2 for each heating phase in TER. The minimum determined for this sensor on the basis of all heating phases is indicated by a transparent ellipse

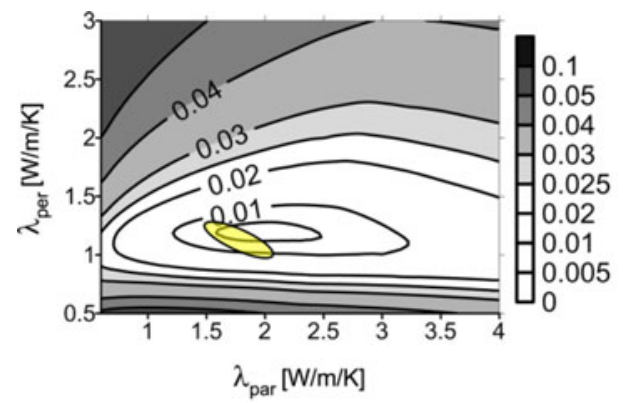

H1.1.
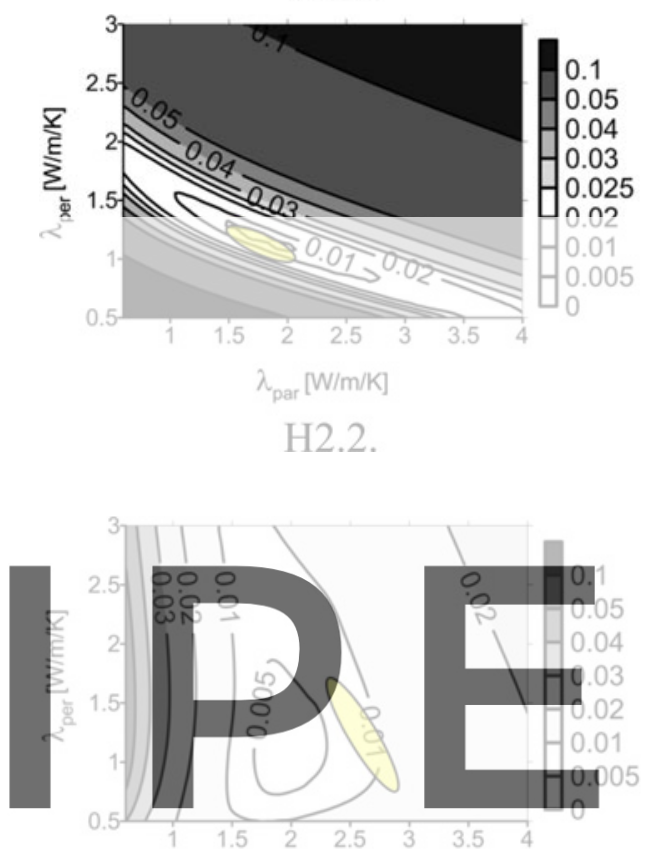

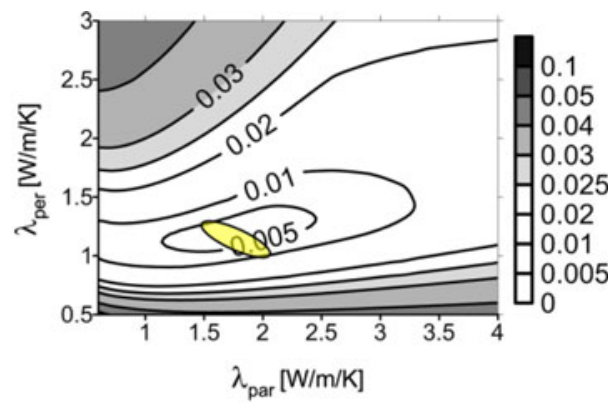

$\mathrm{H} 2.1$.
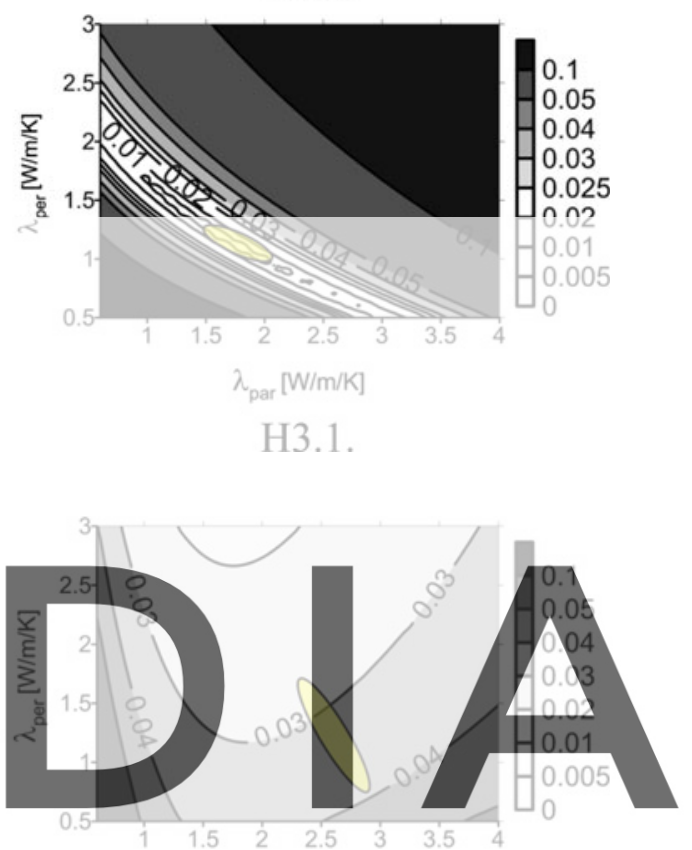

Fig. 6 Map of average relative TER 1203TEM4 for each heating phase in TER. The minimum determined for this

sensor on the basis of all heating

phases is indicated by a parent ellipse H1.1.

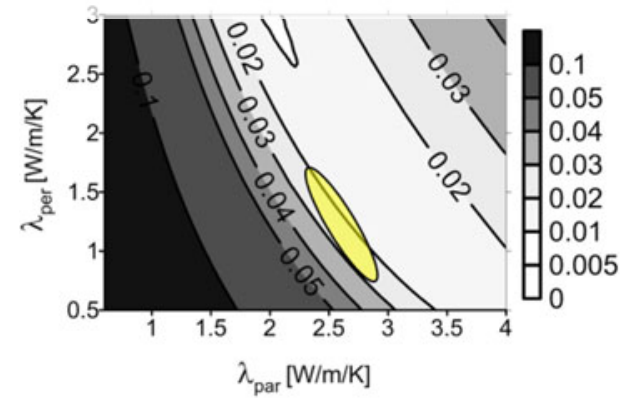

$\mathrm{H} 2.2$.

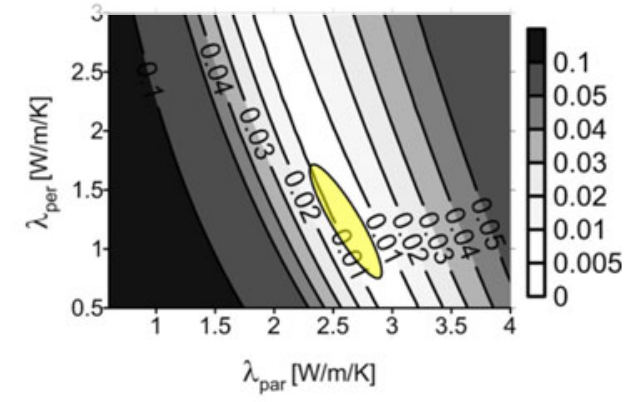

H3.1.
The pairs of thermal conductivity values where $\varepsilon(t)$ is lower than $2 \%$ have been plotted in Fig. 8 for the four synthetic sensors at different times. They correspond to valleys of the difference function encompassing the minimum. Along those valleys, all thermal conductivity pairs are associated with very similar errors, so the determination of the optimum in practice will have a high degree of reliability.
In this synthetic case, for long times, the minimum valleys converge towards a thermal conductivity pair whose product $\lambda_{\text {per }} \cdot \lambda_{\text {par }}=\lambda_{\text {per }}^{*} \cdot \lambda_{\text {par }}^{*}$ for sensors in the bedding plane and to $\lambda_{\text {par }}=\lambda_{\text {par }}^{*}$ for perpendicular sensors. This is related to the fact that the steady-state temperature in parallel and perpendicular sensors is determined by $\sqrt{\lambda_{\text {par }} \cdot \lambda_{\text {per }}}$ and $\lambda_{\text {par }}$, respectively (Table 1 ). This 


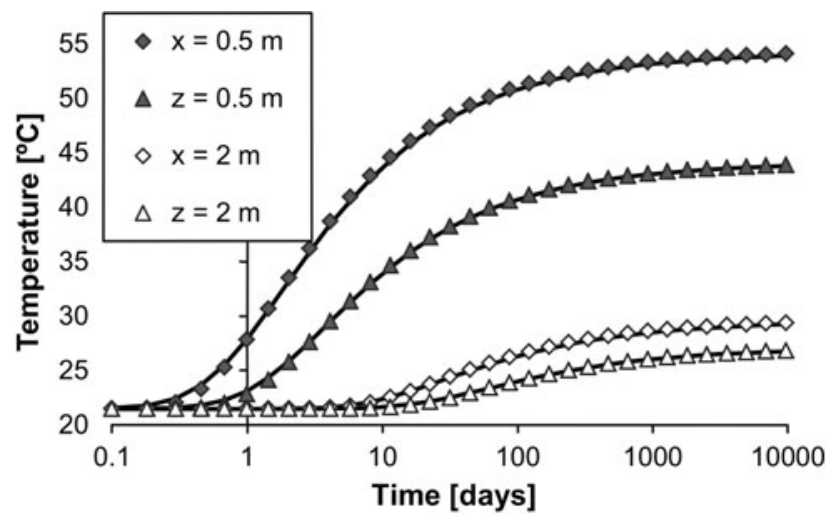

Fig. 7 Comparison of the analytical solution (triangle and diamond dots) against simulation results (full lines) in the bedding plane $(x)$ and in the perpendicular direction $(z)$

convergence occurs more slowly in perpendicular sensors and in farther sensors as the time to reach steady state is inversely proportional to the thermal conductivity between the sensor and the heat source and proportional to the square of the distance between the sensor and the heat source (Table 1). It is noteworthy that the minimum valleys at 1 and 8 days in the sensors at $0.5 \mathrm{~m}$ from the heat source

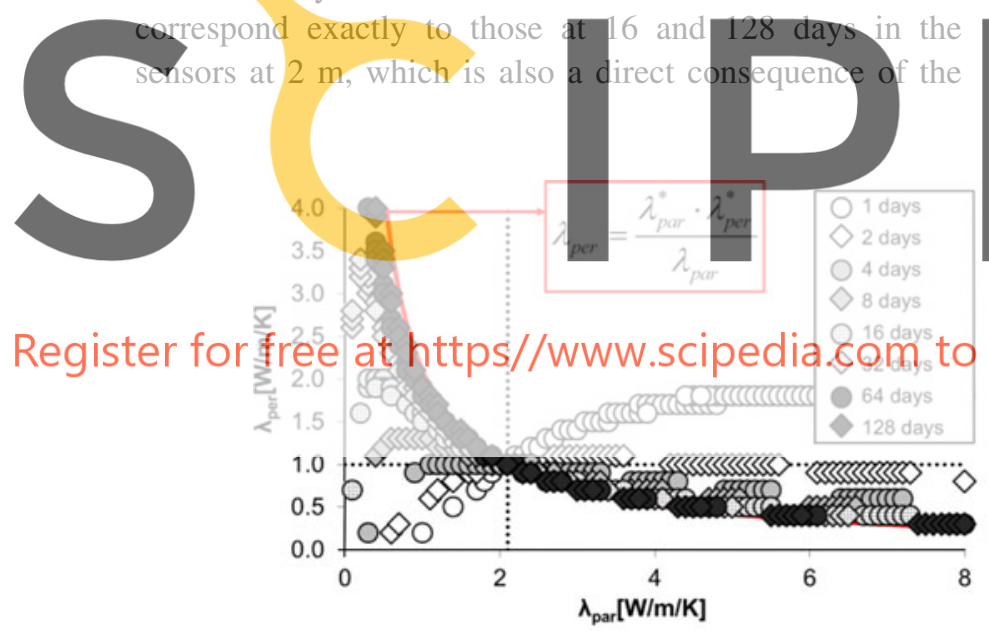

(a)

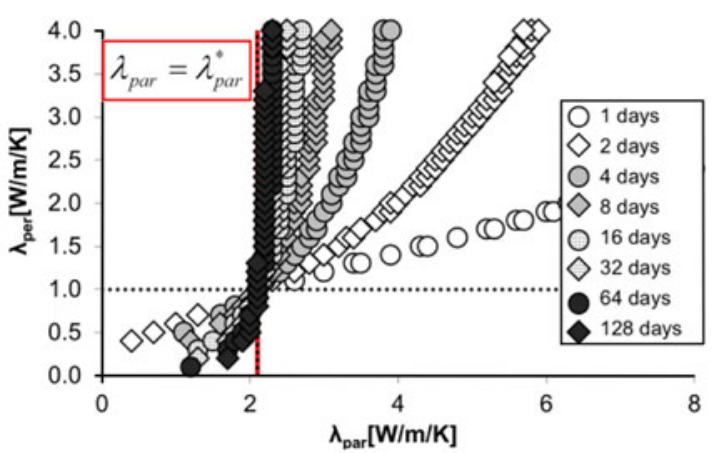

(c) square distance proportionality. It should also be noted that the location of a minimum valley at time $t$ is independent of the applied power.

The analysis of the minimum valleys from Fig. 8 allows the examination of some features of the global analysis presented in the previous section. As a consequence of the indiscriminate time average applied in Eq. (11), relatively long heating phases tend to indicate a minimum valley instead of a single thermal conductivity pair as the information from short times is neglected. This is illustrated in Fig. 9 in which Fig. 8a has been redrawn discarding short times. Without the information from low times, it is not possible to determine the unknown thermal conductivity pair using one single sensor and the time average factor used in the global analysis is thus identified as a potential source of uncertainty. For example, in TER1201TEM2, a parallel sensor relatively close to the heater, long heating periods (H2.2. and H3.1.) indicate a minimum close to the $\lambda_{\text {per }} \cdot \lambda_{\text {par }}=\lambda_{\text {per }}^{*} \cdot \lambda_{\text {par }}^{*}$ valley (Fig. 5). In contrast, shorter heating periods (H1.1. and H2.1.) indicate a clear minimum. Obviously, this problem may be tackled introducing a factor giving more weight to short-term measurements. This solution, however, is difficult to apply in practice as

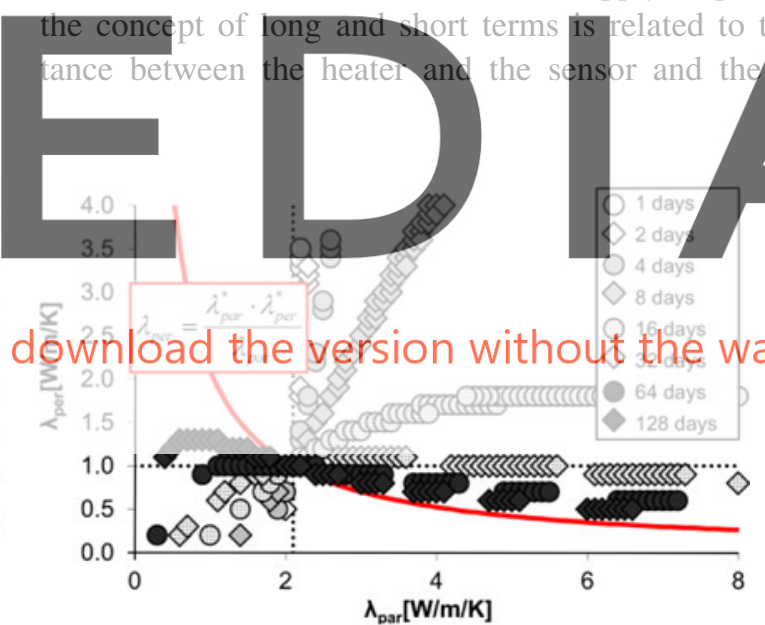

(b)

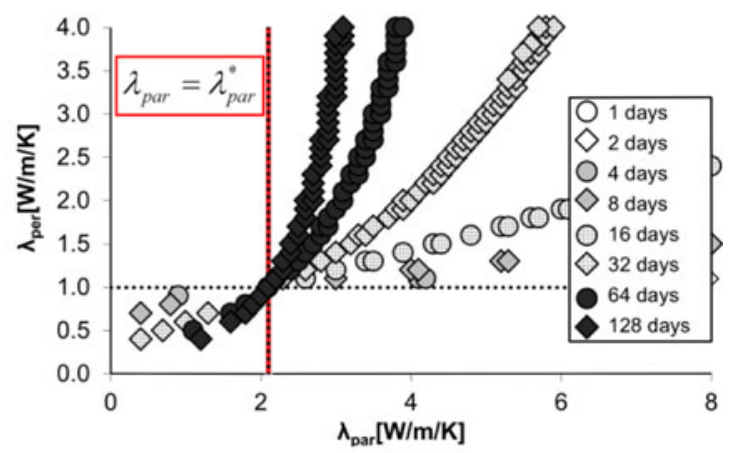

(d)

Fig. 8 Thermal conductivity pairs satisfying $\varepsilon(t)<2 \%$ in the synthetic sensors Par_1 $\left(x^{*}=0.5 \mathrm{~m}\right)(\mathbf{a})$, Par_2 $\left(x^{*}=2 \mathrm{~m}\right)(\mathbf{b})$, Per $\_1\left(z^{*}=0.5 \mathrm{~m}\right)$ (c) and Per_2 $\left(z^{*}=2 \mathrm{~m}\right)(\mathbf{d})$ at different times 


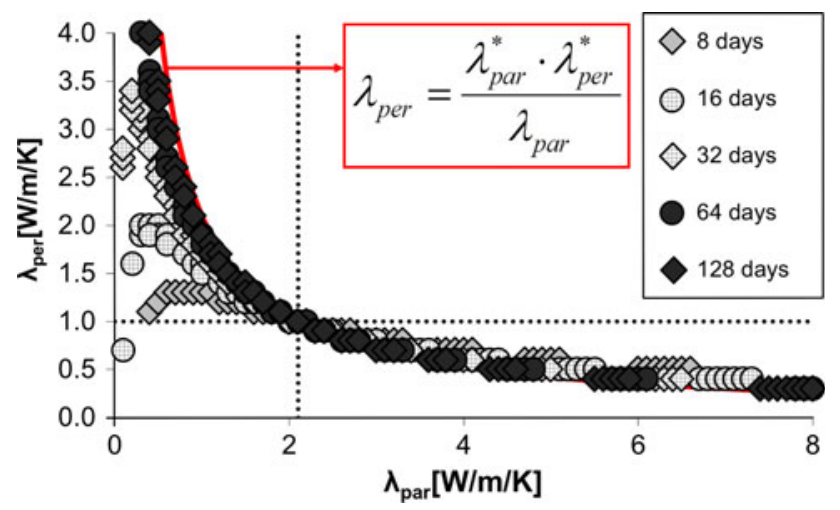

Fig. 9 Thermal conductivity pairs satisfying to $\varepsilon(t)<2 \%$ in synthetic sensor Par_1 $\left(x^{*}=0.5 \mathrm{~m}\right)$ for times $8,16,32,64$ and 128 days

orientation. Unsurprisingly, the same effect is also identified when all heating periods are averaged in each sensor (Fig. 2). Parallel sensors close to the heater (TER1201TEM2, TER1201TEM3 and TER1201TEM4) show an important scatter along the $\lambda_{\text {per }} \cdot \lambda_{\text {par }}=\lambda_{\text {per }}^{*} \cdot \lambda_{\text {par }}^{*}$ valley. Parallel sensors (TER1201TEM1 and TER1201TEM5) further from the heater indicate a single minimum.

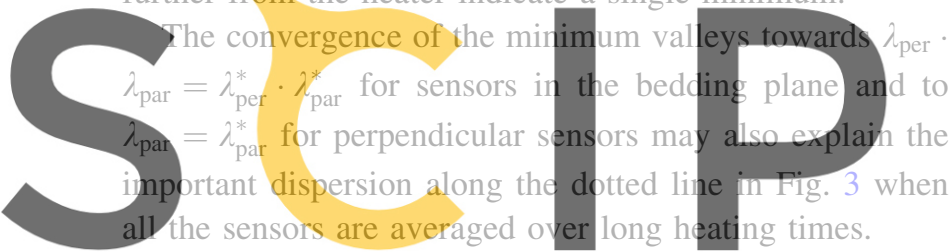

Register for free at https//WwW.scipedia.com to

An evident advantage of a synthetic case is that experi-

mental uncertainties can be simulated easily. According to Eq. (1), three possible experimental uncertainty types may be identified: sensor location uncertainty, power input uncertainty and uncertainty on the temperature measurement itself. Besides, conceptual errors may also be done, in particular on the specific heat, but also on the theoretical assumptions (convection is neglected, medium is considered saturated and changes in porosity are neglected). The theoretical assumptions are considered to be accurate enough. Thermo-Hydro-Mechanical computations performed by Gens et al. (2007) showed that convection was negligible for intrinsic permeability values as high as $10^{-15}$ $\mathrm{m}^{2}$ and that convection started to have influence from values of $10^{-11} \mathrm{~m}^{2}$ (typical for gravels). These values are much beyond the range of argillaceous rocks. It is probable that, after the drilling or during cooling, a zone around the heater desaturates and/or fissures appear. Given the limited extent of this zone and the fact that the experiments are controlled in power, a small impact of temperature on the saturated zone is expected. The impact of each uncertainty on the determination of the best-fitting thermal conductivity pair is now discussed.

\subsubsection{Uncertainty on the Sensor Location}

The emplacement of sensors in an in situ experiment is always subjected to a location uncertainty to some extent. The position of the instrumentation borehole itself is obviously the first uncertainty source. But even in case of accurate knowledge of that position, the location of the sensor within the borehole may also be inaccurate. For example, the diameter of the instrumentation boreholes in the TER experiment is $56 \mathrm{~mm}$. As the instrumentation boreholes are approximately parallel to the heating borehole, emplacing the sensor next to the right or the left wall of the instrumentation borehole is equivalent to a location uncertainty in the direction of the heater of the same order of magnitude as the borehole diameter. It should also be noted that the relevant reference system here is that defined by the heater itself and that a location uncertainty of the heater cannot be discarded either.

A location uncertainty of the same order of magnitude was introduced for the four sensors of the synthetic case:

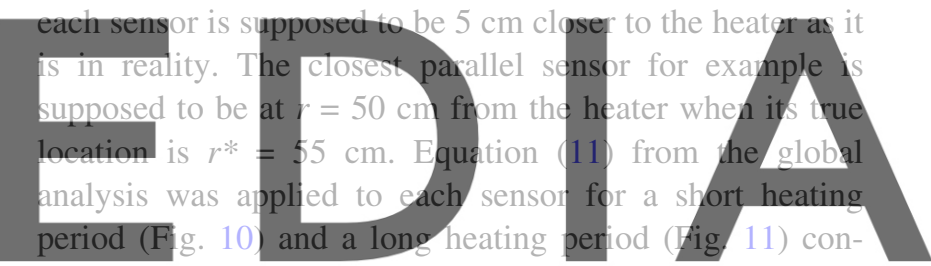
sidering a measurement frequency of 1 day. Most of the sensors indicate a minimum in the long and in the short dpwilopad the versiono without the watermark

thermal conductivity values used in the analysis and a different pair is determined for each sensor. Additionally, some sensors indicate different best-fitting thermal conductivity pair in the short and in the long heating periods. For example, the farthest perpendicular sensor indicates $\lambda_{\text {par }}=1.7 \mathrm{~W} / \mathrm{m} / \mathrm{K}, \lambda_{\text {per }}=0.9 \mathrm{~W} / \mathrm{m} / \mathrm{K}$ in the short heating period and $\lambda_{\text {par }}=2.1 \mathrm{~W} / \mathrm{m} / \mathrm{K}, \lambda_{\text {per }}=0.9 \mathrm{~W} / \mathrm{m} / \mathrm{K}$ in the long heating period. Note that the same phenomenon is observed when the measurement frequency is changed. Other sensors show consistent behaviour between the short and the long heating period, but do not determine the best-fitting thermal conductivity pair precisely in the long heating period. The same type of observations was made in the global analysis of the TER experiment.

The value of the average difference over the examined domain of thermal conductivities should also been discussed. First, the average difference for the best-fitting thermal conductivity pair is not zero and can reach values significantly higher than $2 \%$, for example, in Fig. 10d. This is due to a very large difference for very short times throughout the investigated domain. Secondly, closer 


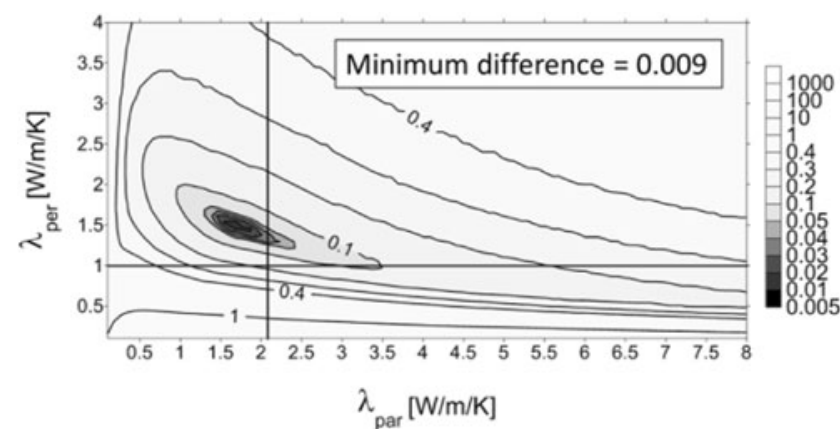

(a)

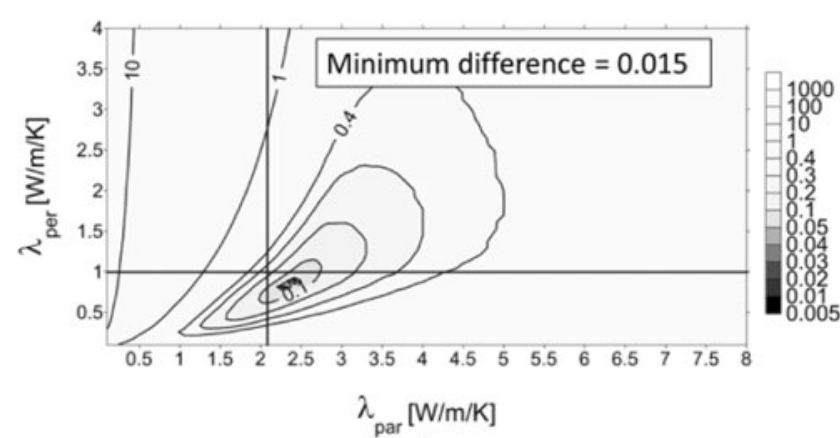

(c)

Fig. 10 Average relative difference maps by applying Eq. (11) for a short heating period (20 days) in sensors at $x^{*}=0.55 \mathrm{~m}$ (a), $x^{*}=2.05$ $\mathrm{m}(\mathbf{b}), z^{*}=0.55 \mathrm{~m}(\mathbf{c})$ and $z^{*}=2.05 \mathrm{~m}(\mathbf{d})$ presuming that the location

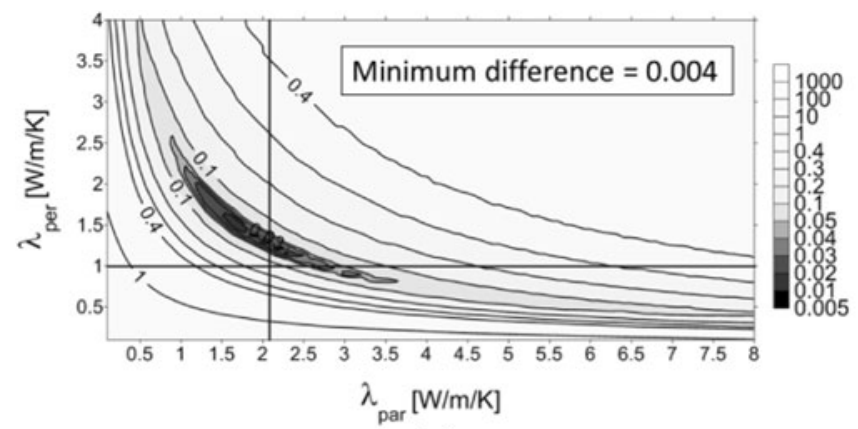

(a)

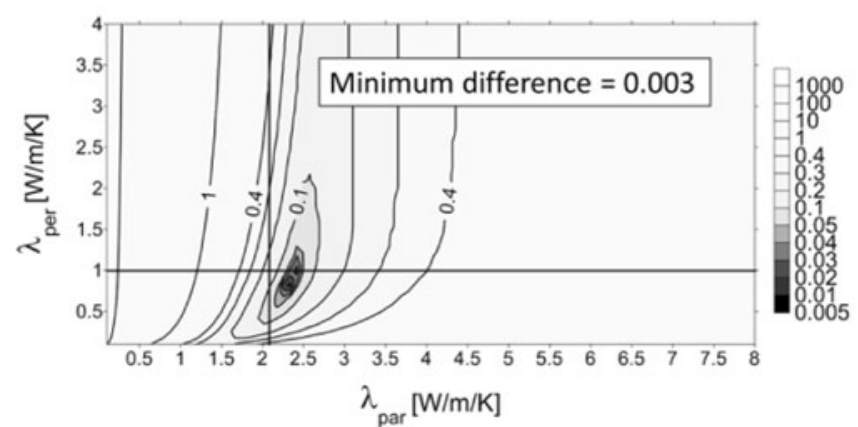

(c)

Fig. 11 Average relative difference maps by applying Eq. (11) for a long heating period (125 days) in sensors at $x^{*}=0.55 \mathrm{~m}(\mathbf{a}), x^{*}=2.05$ $\mathrm{m}(\mathbf{b}), z^{*}=0.55 \mathrm{~m}(\mathbf{c})$ and $z^{*}=2.05 \mathrm{~m}(\mathbf{d})$ presuming that the location

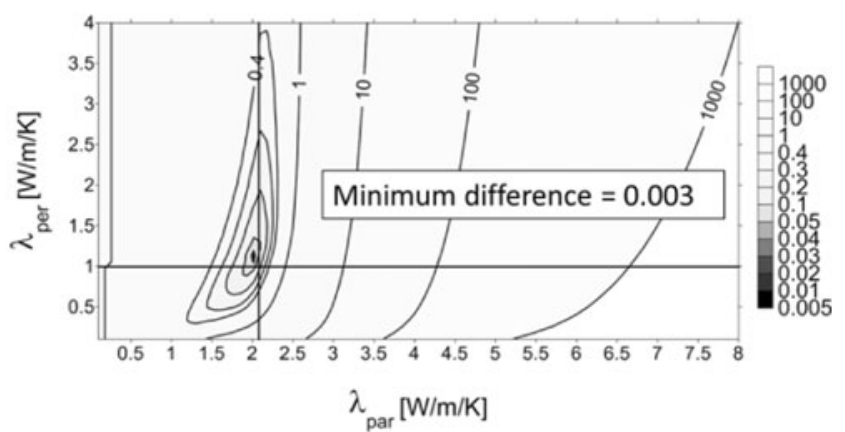

(b)

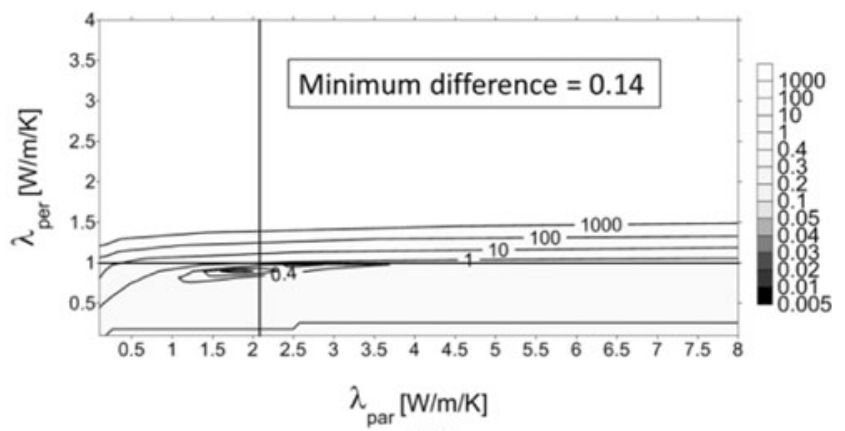

(d)

is $x=0.5 \mathrm{~m} \mathrm{(a),} x=2 \mathrm{~m}(\mathbf{b}), z=0.5 \mathrm{~m} \mathrm{(c)} \mathrm{and} z=2 \mathrm{~m}(\mathbf{d})$. Using measurements at $1,2, \ldots, 20$ days

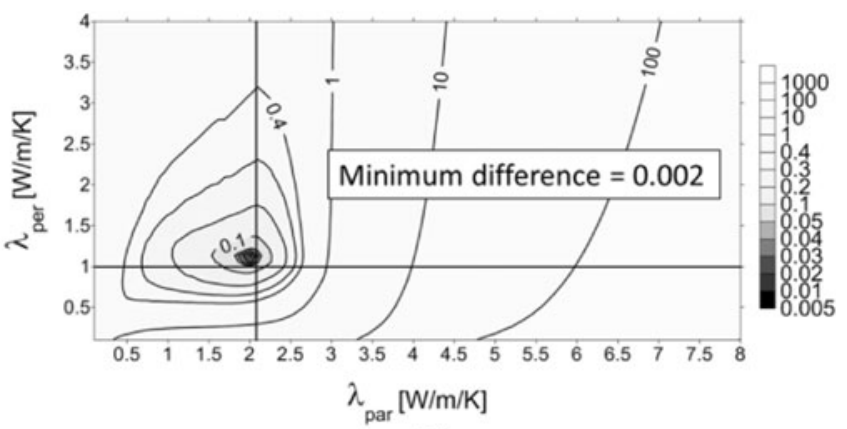

(b)

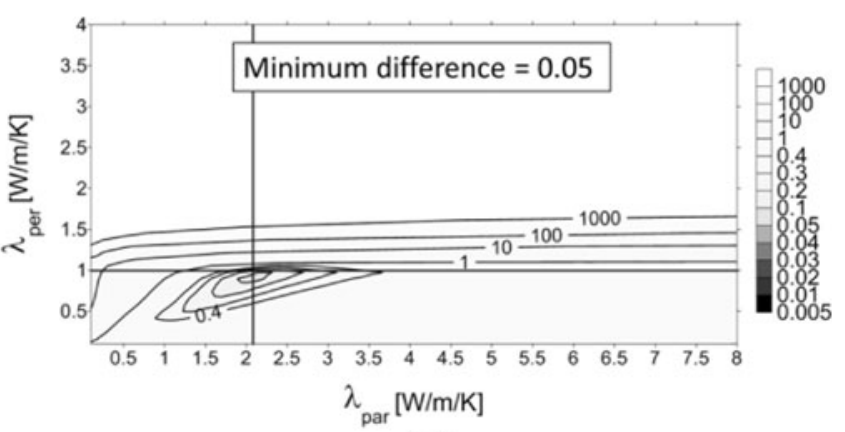

(d)

is $x=0.5 \mathrm{~m}(\mathbf{a}), x=2 \mathrm{~m}(\mathbf{b}), z=0.5 \mathrm{~m}(\mathbf{c})$ and $z=2 \mathrm{~m}(\mathbf{d})$. Using measurements at $1,2, \ldots, 125$ days 


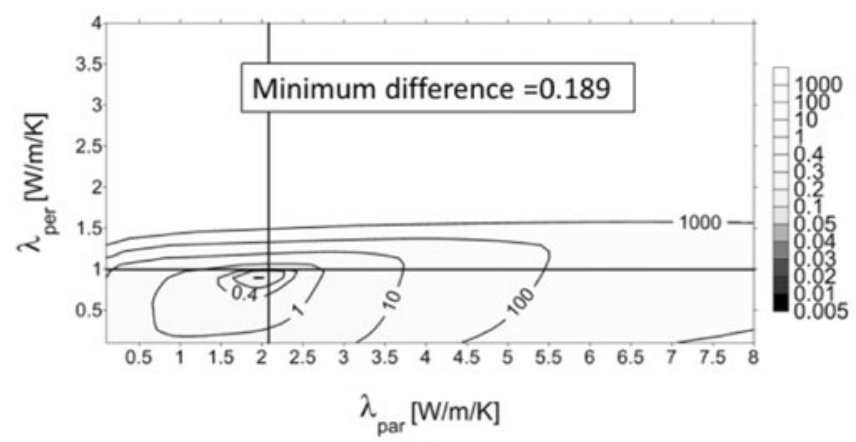

(a)

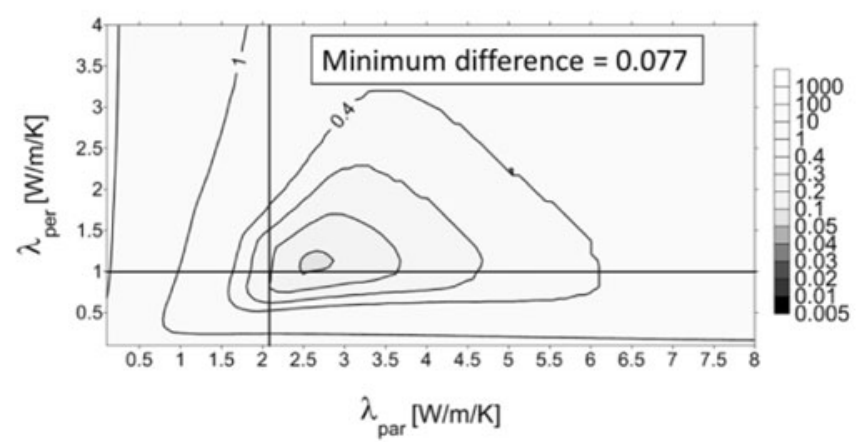

(c)

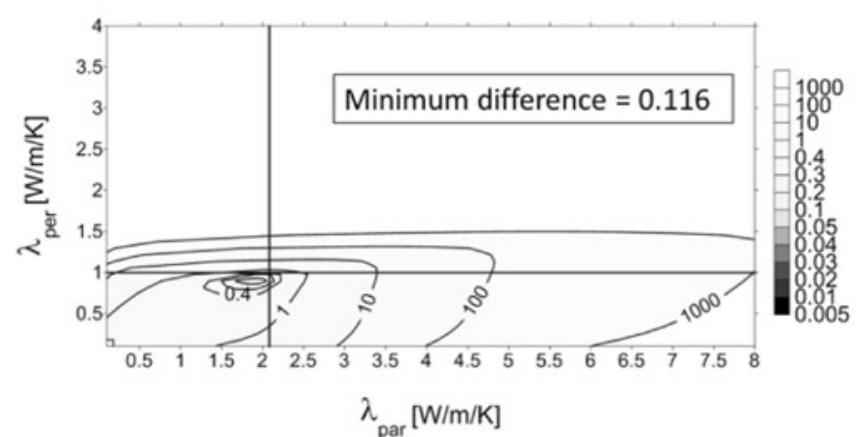

(e)

Fig. 12 Average relative difference maps by applying Eqs. (11) and (13) for a short heating period (a, $\mathbf{c}$ and $\mathbf{e}$ ) and a long heating period (b, $\mathbf{d}$ and $\mathbf{f}$ ) averaging the four sensors (a and $\mathbf{b})$, the two closest

sensors have a relatively low difference all over the domain, typically lower than 1 and the difference in farther sensors increases significantly. For example, the far perpendicular sensor indicates differences over 1,000 for the best-fitting thermal conductivity pair of the closest parallel sensor $\left(\lambda_{\text {par }}=1.75 \mathrm{~W} / \mathrm{m} / \mathrm{K}, \lambda_{\text {per }}=1.5 \mathrm{~W} / \mathrm{m} / \mathrm{K}\right)$.

Having introduced experimental uncertainties, the bestfitting thermal conductivity pair is thus not the same in each sensor and the shape of the difference function away from the minimum will thus influence the results when averaging different sensors. In Fig. 12, the difference maps from the different sensors were averaged using Eq. (13) considering different sensors combinations. It is clear that the contribution from far field sensors spoils the

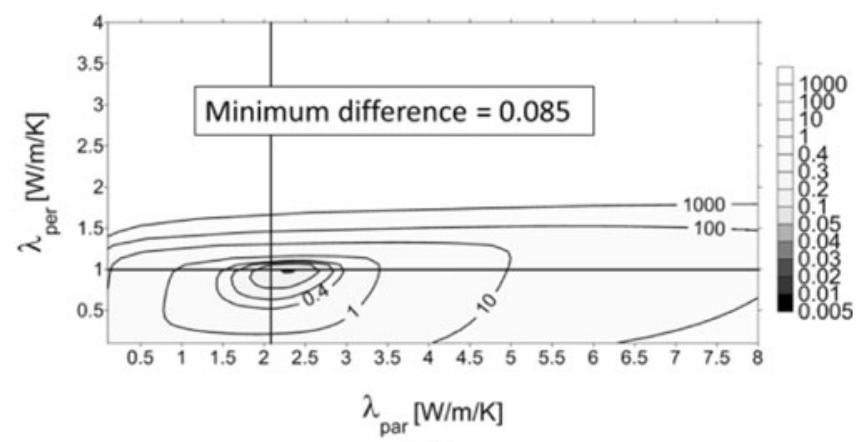

(b)

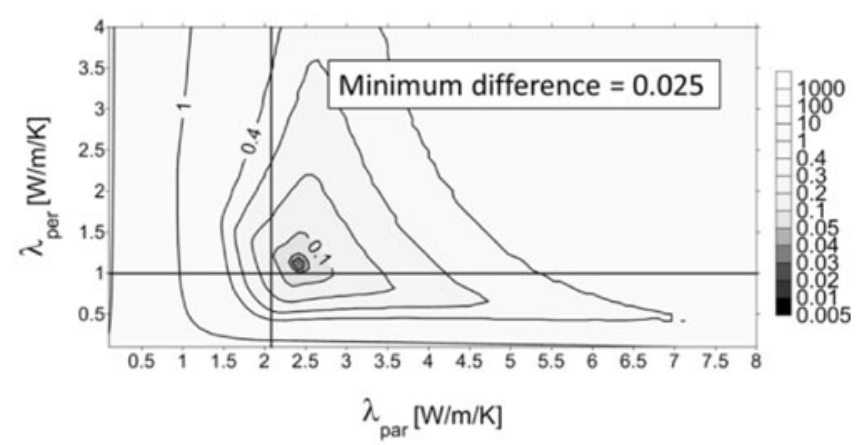

(d)

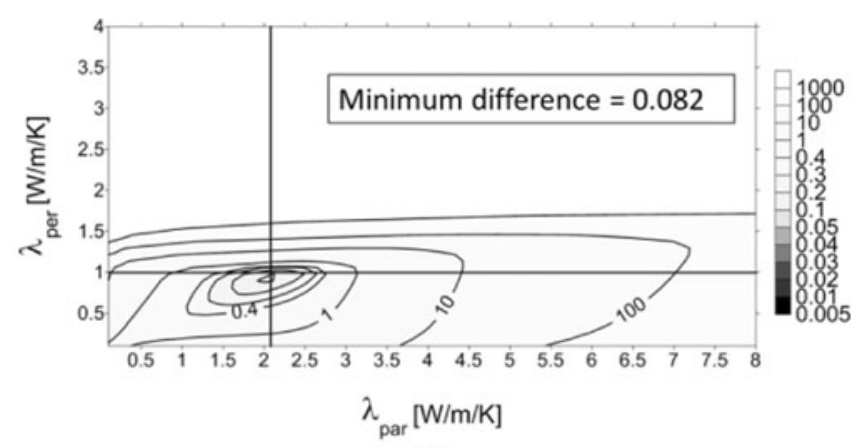

(f)

sensors (c and d) and the two farther sensors (e and f). Using measurements at $1,2, \ldots, 125$ days

information from closer sensors. The difference maps obtained by average of all sensors ( $a$ and $b$ ) are almost identical to those obtained by the average of the two far field sensors (e and f). Moreover, the best-fitting thermal conductivity pair is a function of the duration of the heating period (a vs. b), as it was the case when considering one particular sensor. It is also noteworthy that, when analysed individually, the two closer sensors indicated two different thermal conductivity pairs the average of which was close to the assumed thermal conductivity pair. Applying Eq. (13) to the two closer sensors, another thermal conductivity pair, significantly different from the average of the two individually determined pairs, is obtained (c and d). 
The shape of the difference function was thus identified as a second uncertainty source as it was shown to perform poorly as an average tool. Alternative difference expressions for Eq. (11) were explored. In the first one, the quotient $\Delta T_{\text {real }}$ was replaced by $\Delta T_{\text {sim. }}$. That resulted in even more important overweighting of far field sensors. In the second, alternative absolute difference was used instead of relative difference. That resulted in overweighting close sensors. An attempt was also done to normalise the difference maps for each sensor. That was quickly abandoned as the maximum difference used as a normalising measure is obviously dependent on the size of the investigated thermal conductivity domain and is not intrinsic to the sensor.

Considering the unsatisfactory results from averaging difference maps from different sensors to determine a global average, preference was given to the determination of the best-fitting thermal conductivity pair for each sensor and, then, to compute the average of the thermal conductivity values obtained for individual sensors. To apply this solution, two problems have still to be solved. The first one is the fact that the determined thermal conductivity pair is dependent on the duration of the heating period (e.g. Figs. $10 \mathrm{~d}, 11 \mathrm{~d}$ ). The second one is that, in some cases (close sensors and long heating periods), a minimum valley rather than a single thermal conductivity pair is obtained. Both problems are tackled by applying Eq. (14) as presented in Fig. 9: the thermal conductivity pairs for which the relative difference is lower than $2 \%$ are plotted at different times. Proceeding in this way, the influence of the shape of the difference function away from the minimum is avoided. Accepting only differences lower than $2 \%$ solves the first problem as the very large differences for short times all over the thermal conductivity domain are excluded. We also avoid overweighing long-term measurements in a natural way and a best-fitting thermal conductivity pair is determined independently of the duration of the analysed period.

The parallel and perpendicular thermal conductivity values were determined using the selected methodology for several synthetic sensors in which a known location uncertainty was introduced. The resulting thermal conductivity pairs are shown in Figs. 13 and 14 for sensors at $50 \mathrm{~cm}$ and $2 \mathrm{~m}$, respectively. Diamond symbols indicate sensors in the bedding plane, circle symbols sensors in the perpendicular direction and rectangular symbols sensors in an intermediate orientation. Iso-curves of equivalent thermal conductivity $\left(\lambda_{0}\right)$ and anisotropy ratio $(\alpha)$ have been added to these figures. Independently of the magnitude or the direction of the location uncertainty, the determined thermal conductivity pairs lie on the same equivalent thermal conductivity iso-curve. A significant scatter of the determined anisotropy ratio is, however, found. This observation

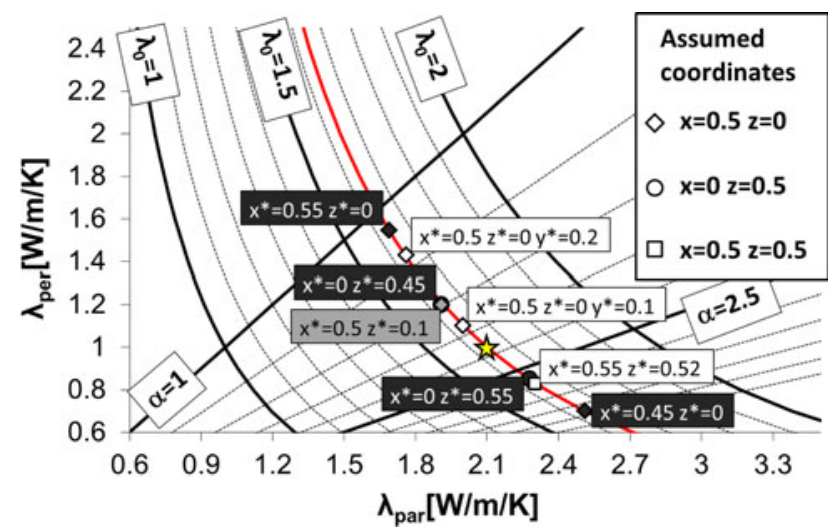

Fig. 13 Influence of location uncertainties on the determination of the thermal conductivity pair for sensors at $50 \mathrm{~cm}$. The five-branch star indicates the pair used in the synthetic case

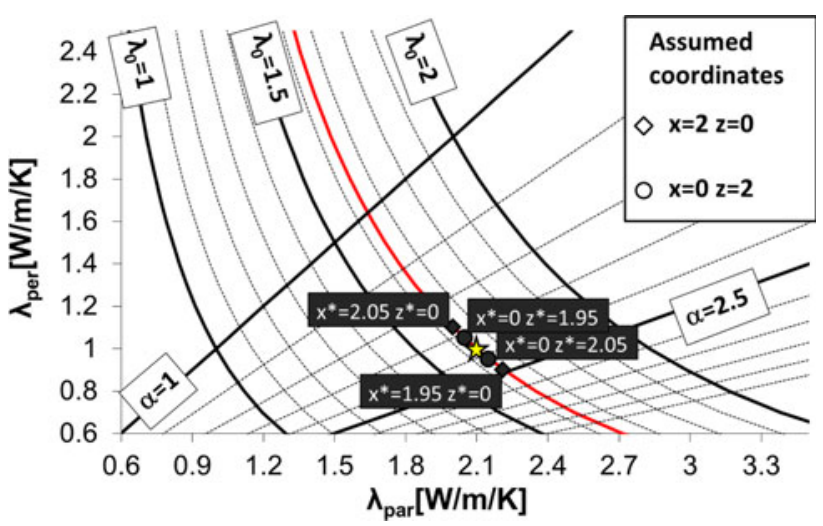

Fig. 14 Influence of location uncertainties on the determination of the thermal conductivity pair for sensors at $2 \mathrm{~m}$. The five-branch star indicates the pair used in the synthetic case

suggests that it may be preferable to characterise the thermal conductivity in terms of equivalent thermal conductivity and anisotropy ratio instead of thermal conductivity in the bedding plane and in the perpendicular direction. In Table 6 , the determined thermal conductivity values for the sensors used in Figs. 13 and 14 are reported and the average is calculated. Obviously, the average of the equivalent thermal conductivity is that obtained for every sensor. It is

Table 6 Determined thermal conductivity values for four synthetic sensors with a $5 \mathrm{~cm}$ localisation uncertainty

\begin{tabular}{lllll}
\hline Sensor & $\lambda_{\text {par }}$ & $\lambda_{\text {per }}$ & $\lambda_{0}$ & $\lambda_{h} / \lambda_{v}$ \\
\hline$x^{*}=0.55 \mathrm{~m} \mathrm{\&} z^{*}=0$ & 1.69 & 1.55 & 1.64 & 1.09 \\
$x^{*}=2.05 \mathrm{~m} \& z^{*}=0$ & 2.00 & 1.10 & 1.64 & 1.82 \\
$x^{*}=0 \& z^{*}=0.55 \mathrm{~m}$ & 2.28 & 0.85 & 1.64 & 2.68 \\
$x^{*}=0 \& z^{*}=2.05 \mathrm{~m}$ & 2.15 & 0.95 & 1.64 & 2.26 \\
Average & 2.03 & 1.11 & 1.64 & 1.96 \\
Calculated from average of $\lambda_{0}$ and $\lambda_{h} / \lambda_{v}$ & 2.05 & 1.05 & & \\
\hline
\end{tabular}


noteworthy that using the average equivalent thermal conductivity and the average anisotropy ratio to calculate thermal conductivity values in the bedding and in the perpendicular direction yields better results than averaging the bedding and perpendicular values directly.

\subsubsection{Uncertainty on the Power Input}

An uncertainty on the power input can occur in heating experiments (Gens et al. 2009). In practice, the applied power is not measured directly. In fact, we impose an electrical current from the gallery. What is really injected at the heater depends on potential losses during transport. Those losses can be estimated but they depend on a number of factors, e.g. the temperature of the electrical line. The structure of the heater itself can also induce some uncertainty and will probably result in applying a non-uniform power along its length. This can be caused by metallic ends that would be equivalent to a larger heater length or irregularities of the wire spirals around the heating core.

The effect of over- or underestimation of the applied power has been summarised in Fig. 15 and it is quite remarkable. Independently of the magnitude of the power input uncertainty, sensors in the bedding plane provide an exact estimation of the perpendicular thermal conductivity and perpendicular sensors provide an exact estimation of the parallel thermal conductivity. Sensors at different distances from the heat source, but in the same orientation, provide the same best-fitting thermal conductivity pair. For the same power input uncertainty, the determined equivalent thermal conductivity is the same in both directions. In fact it can be directly approximated from Eq. (1) using the steady-state factor:

$\delta \lambda_{0}=\frac{-\lambda_{0} \cdot \delta q}{q+\delta q}$

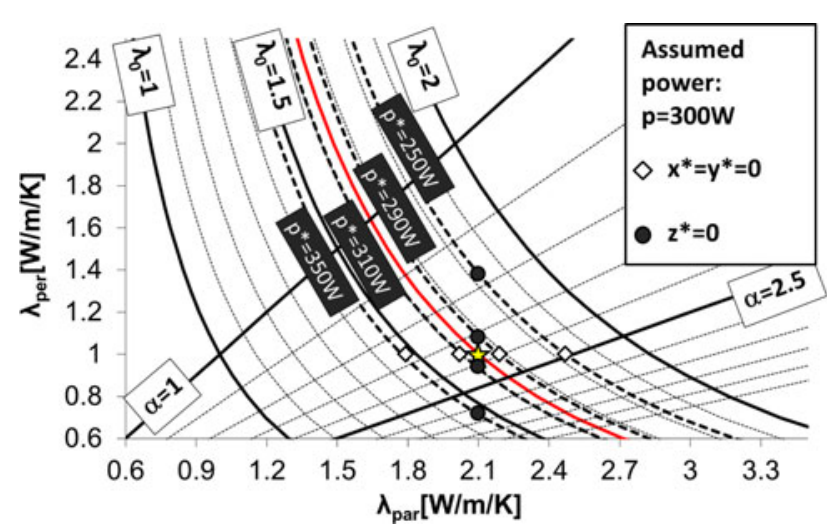

Fig. 15 Influence of power input uncertainties on the determination of the thermal conductivity pair. The five-branch star indicates the pair used in the synthetic case
It is apparent that an uncertainty on the power input will induce a difference between the thermal conductivities determined from perpendicular and parallel sensors. This feature will be used in the determination methodology.

\subsubsection{Uncertainty on the Temperature Measurements}

Temperature measurement uncertainties may obviously be related directly to the temperature sensor itself. Sensor manufacturers estimate a minimum temperature uncertainty of about $0.1{ }^{\circ} \mathrm{C}$. Additionally, a possible uncertainty of about $0.2-0.3{ }^{\circ} \mathrm{C}$ (Wileveau 2005) is estimated. Another indirect uncertainty source on the temperature measurement may occur in the case that the boundary conditions of the problem (e.g. temperature variation in nearby galleries) are not reproduced adequately. This second uncertainty source is illustrated in Fig. 16 in which the temperature evolution measured in two sensors of the TER experiment has been plotted. Both sensors lie at the same distance from the heating device, but one is relatively close to a gallery and the other one is relatively far. The results from three simulations have been added to illustrate the influence of temperature variations on the gallery. In the first computation, only the heater was taken into account and obviously the simulation predicts a very similar temperature evolution in the two sensors that differ significantly from the measurements. The difference between this simulation and the measurements is explained by the second simulation that considers exclusively the temperature variation in the gallery (no heating is applied at the heater boundary). Indeed, if the two effects are taken into account in the simulation (the third simulation), a good agreement between the simulation and the measurements is obtained.

As for the other uncertainty sources, an attempt was done to quantify the effect of temperature measurement uncertainties (Fig. 17). Two constant errors on the temperature measurement were applied: 0.5 and $1.5^{\circ} \mathrm{C}$. The

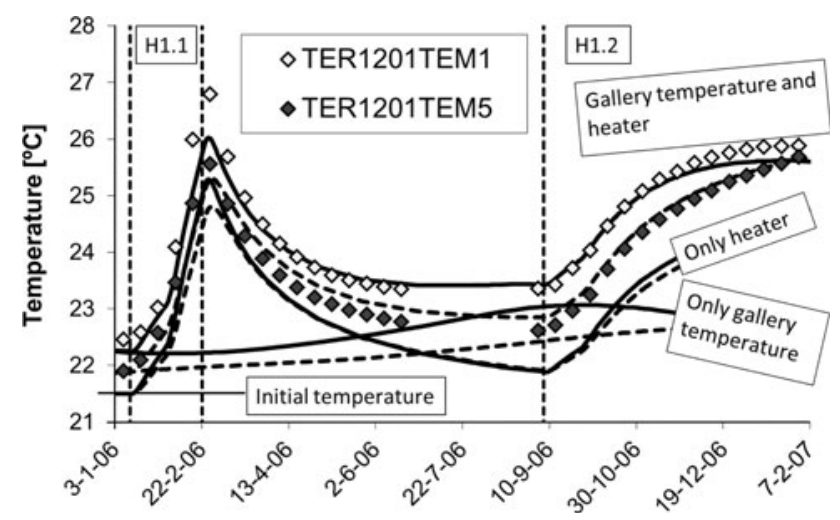

Fig. 16 Measured temperature evolution in TER1201TEM1 and TER1201TEM5. Simulation results have been added for three simulations (full lines for TER1201TEM1 and dotted lines for TER1201TEM5) 


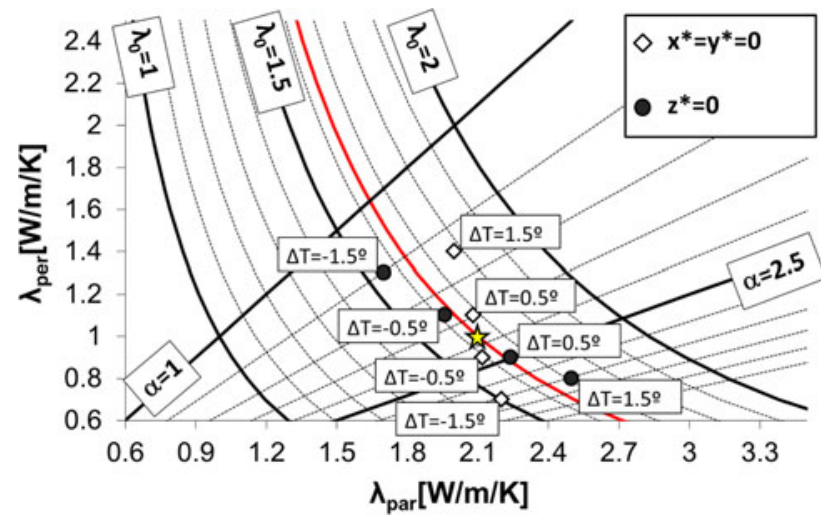

Fig. 17 Influence of temperature measurement uncertainties on the determination of the thermal conductivity pair. The five-branch star indicates the pair used in the synthetic case

first one can be considered as an upper bound for direct sensor uncertainties and the second one as an upper bound for indirect uncertainties. Even if the uncertainty is moderate, a significant difference from the thermal conductivity pair used in the calculation is observed. As in the location uncertainty case, the scatter of the anisotropy ratio is much larger than that of the equivalent thermal conductivity.

\subsubsection{Uncertainty on the Specific Heat}

We already commented that the uncertainty on the specific heat is much lower than the uncertainty on the thermal conductivity as it does not depend on the grain arrangement but only on the volumetric composition of the porous medium. Nevertheless, it is reasonable to expect a spatial variation of the specific heat as the medium composition can vary as a function of the sedimentary level. That spatial variation cannot be taken into account in the finite element computation due to the lack of information on the spatial variability of the porous medium composition. A possible variation of the specific heat of $100 \mathrm{~J} / \mathrm{kg} / \mathrm{K}$, which is probably exaggerated, was investigated in Fig. 18. The main findings may be summarised as follows:

- An uncertainty on the specific heat induces a constant uncertainty on the equivalent thermal conductivity independently of the sensor orientation.

- The uncertainty induced on the thermal conductivity anisotropy is dependent on the sensor orientation and may reach important values for some sensor orientations.

- Sensors at different distances from the heat source but along the same orientation provide the same best-fitting thermal conductivity pair.

- Parallel sensors indicate a best-fitting thermal conductivity pair on $\lambda_{\text {per }} \cdot \lambda_{\text {per }} \cdot \lambda_{\text {par }}=\lambda_{\text {per }}^{*} \cdot \lambda_{\text {par }}^{*}$ and perpendicular sensors on $\lambda_{\text {par }}=\lambda_{\text {par }}^{*}$ as the steady-state temperature is not influenced by the specific heat.

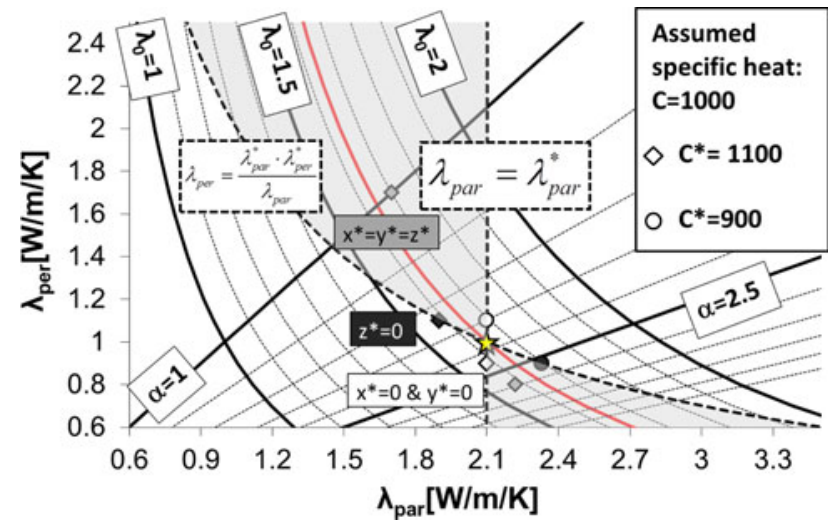

Fig. 18 Influence of specific heat uncertainties on the determination of the thermal conductivity pair. The five-branch star indicates the pair used in the synthetic case

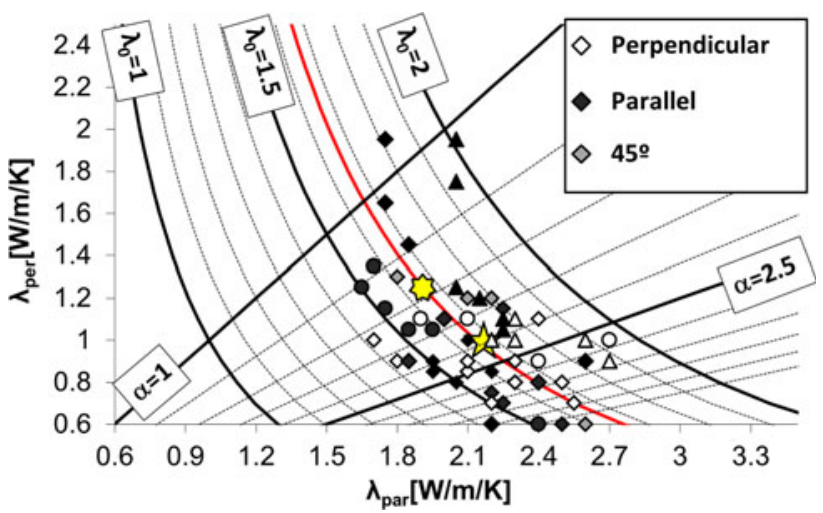

Fig. 19 Best-fitting thermal conductivity pairs in each sensor for each heating level in the TER experiment. The seven-branch star indicates the mean of laboratory measurements and the five-branch star the average of in situ determined pairs

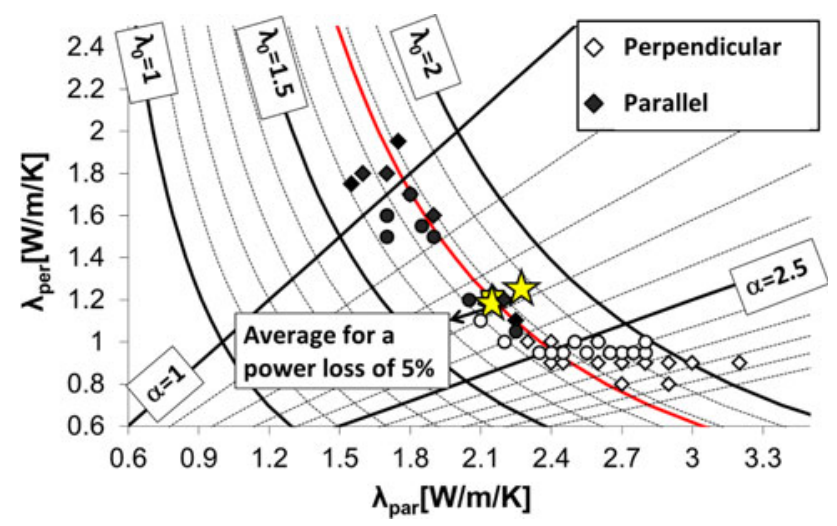

Fig. 20 Best-fitting thermal conductivity pairs in each sensor for each heating level in the HE-D experiment. The seven-branch star indicates the mean of laboratory measurements. The five-branch stars indicate the average of in situ determined pairs as explained in determination methodology 


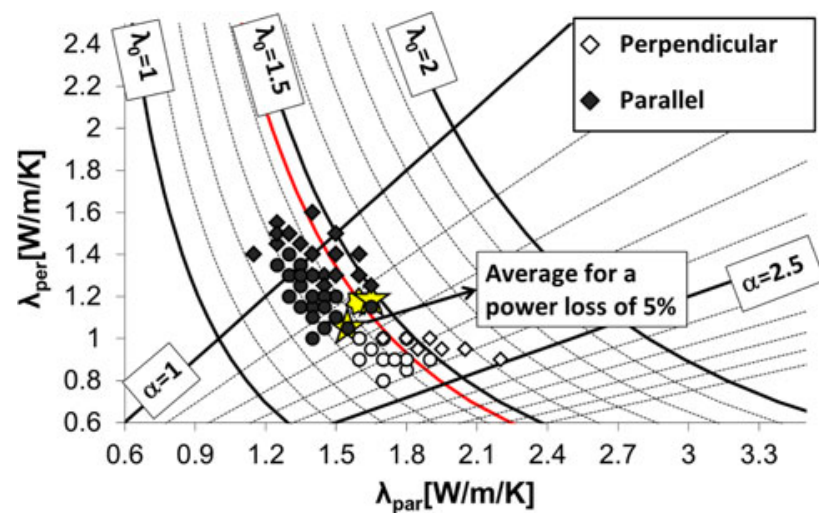

Fig. 21 Best-fitting thermal conductivity pairs in each sensor for each heating level in the ATLAS experiment. The seven-branch star indicates the mean of laboratory measurements. The five-branch stars indicate the average of in situ determined pairs as explained in determination methodology

- As a direct consequence of the last point, all best-fitting thermal conductivity pairs will fall in the area between the two extreme minimum valleys indicated by a grey background in the figure.

It is important to emphasise that, in a similar way as for localisation and power uncertainties, specific heat uncertainty induces a small uncertainty on the equivalent thermal conductivity.

\subsection{Determination Methodology}

As a result of the above considerations, the following methodology for the determination of the anisotropic thermal conductivity is adopted:
- 3D computations of the considered experiment are run for different thermal conductivity pairs covering a wide range (beyond the natural variation of thermal conductivity).

- Values of thermal conductivity are obtained from each individual sensor and for each heating state. The determination of the pair of thermal conductivity values is performed from the intersection of lowdifference valleys plotted at different times (as in Fig. 8).

- This is done whenever possible, but some sensors and/ or some heating phases provide less good quality data, probably as a consequence of the above-described uncertainties. In those cases, the determination methodology fails and the sensor is excluded. This is one of the advantages of the present method in comparison with a more general approach. In the general case, a bath quality sensor is included and it perturbs the determination.

- An overall value of thermal conductivities is obtained from the average of all the sensors. If there are quite different numbers of sensors aligned parallel and perpendicular to the bedding planes, the average of each orientation will be computed first and a final overall value will be obtained from the average of the sensors at the two different orientations.

- If there is a significant difference between the thermal conductivities computed from sensors parallel and perpendicular to bedding planes, it will be taken as an indication of uncertainty in the heating power that will be adjusted accordingly.
Fig. 22 Best-fitting thermal conductivity pairs in each sensor in the TED experiment for H1.1. The seven-branch star indicates the mean of laboratory measurements and the fivebranch star the average of in situ determined pairs

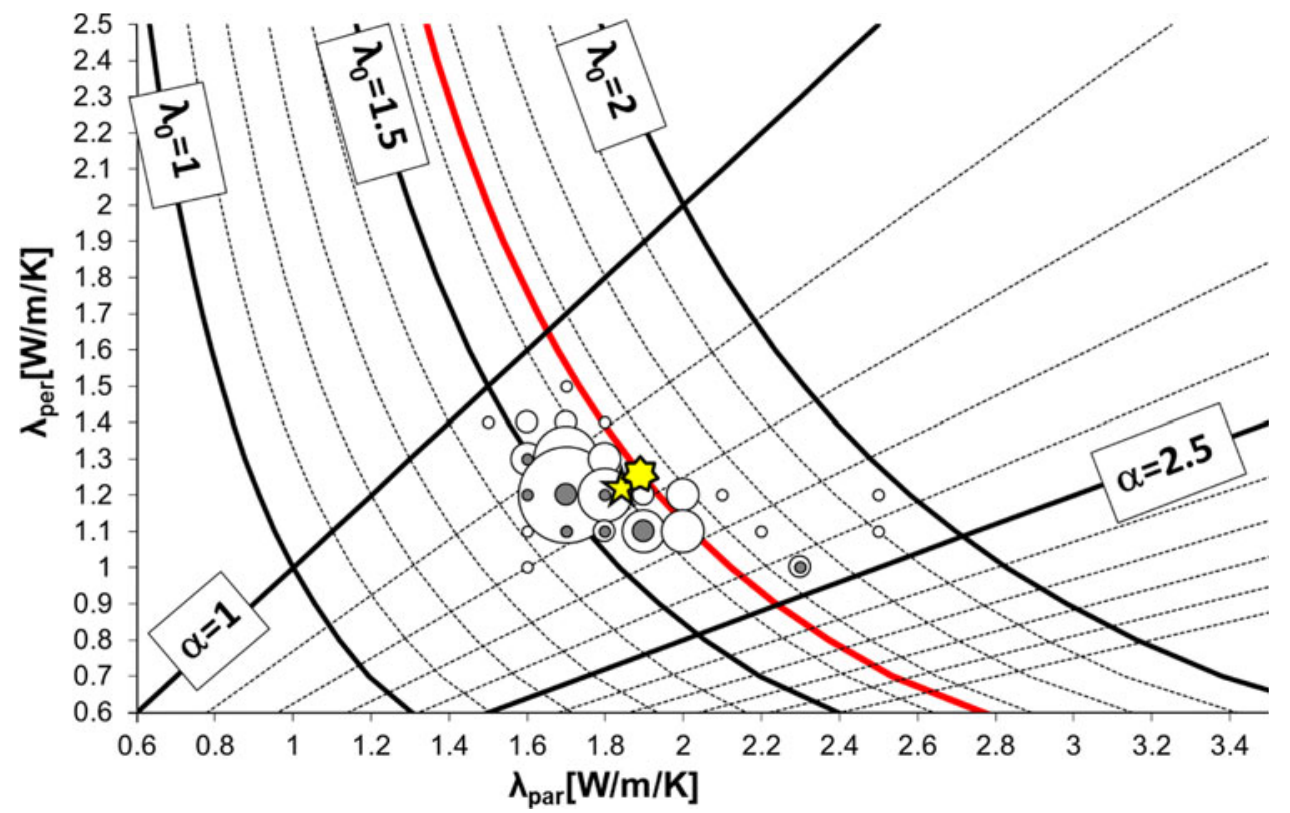




\section{Application to Four In Situ Heating Tests}

The proposed methodology has been applied to the four heating in situ tests. A 3D thermal conceptual model was thus built for each of the experiments. In general, we considered:

- A domain extension limited by the adjacent geological units in vertical direction and by the presence of other galleries (or about $50 \mathrm{~m}$ when no gallery was present) in horizontal direction.

- Prescription of the initial temperature on top and bottom boundaries.

- Null heat flux on the vertical limits and the measured temperature on the gallery walls since their excavation.
- Application of power on the heater walls corresponding with the really applied one. The exact position of the heater was considered.

- The mesh size was typically 50.000 to 200.000 nodes depending on the problem complexity.

The obtained results are shown in Fig. 19 (TER experiment), Fig. 20 (HE-D experiment), Fig. 21 (ATLAS experiment) and Figs. 22, 23 and 24 (TED experiment).

The best-fitting thermal conductivity pairs for each sensor in each heating phase of the TER experiment are presented in Fig. 19. Full symbols stand for sensors in the bedding plane and blank symbols for perpendicular sensors. Although some parallel sensors indicate values close
Fig. 23 Best-fitting thermal conductivity pairs in each sensor in the TED experiment for H1.2. The seven-branch star indicates the mean of laboratory measurements and the fivebranch star the average of in situ determined pairs
Fig. 24 Best-fitting thermal conductivity pairs in each sensor in the TED experiment for H1.3. The seven-branch star indicates the mean of laboratory measurements and the fivebranch star the average of in situ determined pairs
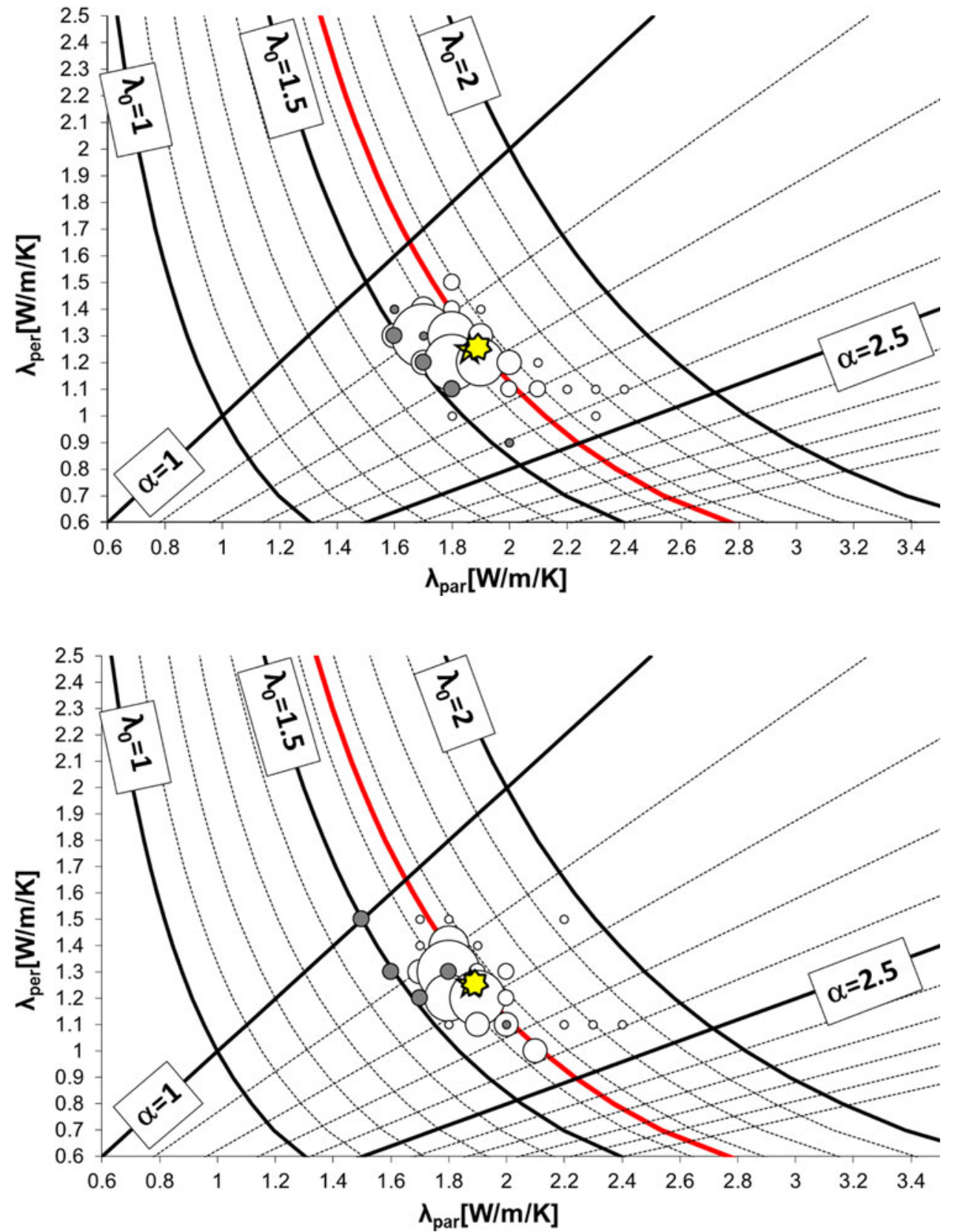
to isotropy and that perpendicular sensors tend to provide a better discrimination of the perpendicular thermal conductivity, no clear trend is observed. The average value was thus calculated on the basis of all sensors and all heating phases. It is striking that the average equivalent thermal conductivity is very similar to the equivalent thermal conductivity determined in the laboratory (samples from the TER area). However, for the anisotropy ratio, a significant scatter is observed. The standard deviation calculated for the anisotropy ratio $(0.88)$ is thus more significant than the standard deviation of the equivalent thermal conductivity $(0.14 \mathrm{~W} / \mathrm{m} / \mathrm{K})$.

In the HE-D (Fig. 20) and the ATLAS experiments, (Fig. 21), the parallel sensors and the perpendicular sensors tend to indicate two different thermal conductivity pairs. Some power loss was assumed as a possible explanation for this kind of pattern and a second analysis was performed for those two tests. Diamond dots indicate the analysis in which no power loss was applied and circle dots indicate the best-fitting thermal conductivity pair determined in an analysis considering a power loss of $5 \%$. Due to the differences between the two sensor groups and to the fact that more perpendicular sensors were available in the HE-D and more parallel ones in the ATLAS, the global average is not done on all sensors directly as indicated above. The averages of the perpendicular sensors and of the parallel sensors are done first. The two obtained averaged thermal conductivity pairs are then used to calculate what we called the final average. A direct consequence of this is that the standard deviation can only be computed for the equivalent thermal conductivity.

Because of the important number of sensors in the TED experiment, the results are presented in three different Figs. 22, 23 and 24 (one for each of the analysed heating phases). In these figures, the size of the dots is proportional to the number of sensors for which that particular best-fitting thermal conductivity pair has been obtained. No consistent differences between the results from parallel and perpendicular sensors were observed.

The thermal conductivity values determined in the laboratory and in the field are summarised in Table 7. Wherever possible, a standard deviation was indicated. The case of the TER and TED experiments is worth commenting. In both cases, the determined equivalent thermal conductivity value is very similar not only between the two tests but also when they are compared to laboratory values. For the determined anisotropy ratio, significant differences were found for the in situ TER experiment. In the TER experiment, important uncertainties (particularly on the sensors location) were found. In accordance with the findings about the impact of experimental uncertainties, those uncertainties seem to influence more of the anisotropy ratio than the equivalent thermal conductivity. The TED experiment was performed after the TER experiment
Table 7 Summary of the thermal conductivity values determined in the laboratory and in the in situ heating tests for the three host rocks

\begin{tabular}{|c|c|c|c|c|}
\hline & $\lambda_{\text {par }}(\mathrm{W} / \mathrm{m} / \mathrm{K})$ & $\lambda_{\text {per }}(\mathrm{W} / \mathrm{m} / \mathrm{K})$ & $\lambda_{0}(\mathrm{~W} / \mathrm{m} / \mathrm{K})$ & $\lambda_{\text {par }} / \lambda_{\text {per }}$ \\
\hline \multicolumn{5}{|l|}{ Boom Clay } \\
\hline \multicolumn{5}{|l|}{ ATLAS } \\
\hline Average & $1.55^{\mathrm{a}}$ & $1.06^{\mathrm{a}}$ & 1.35 & 1.46 \\
\hline SD & & & 0.05 & \\
\hline \multicolumn{5}{|l|}{ Lab } \\
\hline Average & 1.60 & 1.18 & 1.44 & 1.36 \\
\hline \multicolumn{5}{|l|}{ SD } \\
\hline \multicolumn{5}{|c|}{ Callovo-Oxfordian Clay } \\
\hline \multicolumn{5}{|l|}{ TER } \\
\hline Average & 2.17 & 1.00 & 1.65 & 2.40 \\
\hline SD & 0.28 & 0.30 & 0.14 & 0.88 \\
\hline \multicolumn{5}{|l|}{ TER lab } \\
\hline Average & 1.91 & 1.25 & 1.66 & 1.53 \\
\hline \multicolumn{5}{|l|}{ SD } \\
\hline \multicolumn{5}{|l|}{ TED1.1. } \\
\hline Average & 1.84 & 1.23 & 1.60 & 1.52 \\
\hline SD & 0.21 & 0.11 & 0.10 & 0.28 \\
\hline \multicolumn{5}{|l|}{ TED1.2. } \\
\hline Average & 1.86 & 1.25 & 1.63 & 1.51 \\
\hline $\mathrm{SD}$ & 0.17 & 0.12 & 0.07 & 0.27 \\
\hline \multicolumn{5}{|l|}{ TED1.3. } \\
\hline Average & 1.88 & 1.26 & 1.64 & 1.51 \\
\hline $\mathrm{SD}$ & 0.17 & 0.13 & 0.08 & 0.27 \\
\hline \multicolumn{5}{|l|}{ TED lab } \\
\hline Average & 1.92 & 1.27 & 1.67 & 1.51 \\
\hline SD & 0.05 & 0.04 & & \\
\hline \multicolumn{5}{|c|}{ Opalinus Clay } \\
\hline \multicolumn{5}{|l|}{ HE-D } \\
\hline Average & $2.15^{\mathrm{a}}$ & $1.19^{\mathrm{a}}$ & 1.80 & 1.81 \\
\hline SD & & & 0.09 & \\
\hline \multicolumn{5}{|l|}{ Lab } \\
\hline $\begin{array}{l}\text { Average } \\
\text { SD }\end{array}$ & 2.15 & 1.2 & 1.77 & 1.79 \\
\hline
\end{tabular}

${ }^{a}$ Corrected average values (average of the parallel sensors and perpendicular sensors considering a power loss of $5 \%$ )

Table 8 Proposed reference thermal conductivity values for the three rocks

\begin{tabular}{lllll}
\hline & $\begin{array}{l}\lambda_{\text {par }} \\
(\mathrm{W} / \mathrm{m} / \mathrm{K})\end{array}$ & $\begin{array}{l}\lambda_{\text {per }} \\
(\mathrm{W} / \mathrm{m} / \mathrm{K})\end{array}$ & $\begin{array}{l}\lambda_{0} \\
(\mathrm{~W} / \mathrm{m} / \mathrm{K})\end{array}$ & $\lambda_{\text {par }} / \lambda_{\text {per }}$ \\
\hline Boom Clay & 1.55 & 1.06 & 1.35 & 1.46 \\
Callovo-Oxfordian Clay & 1.88 & 1.26 & 1.64 & 1.5 \\
Opalinus Clay & 2.15 & 1.2 & 1.8 & 1.8 \\
\hline
\end{tabular}

to reduce these uncertainties and to verify the agreement between thermal conductivity values measured in the laboratory and in situ among other reasons. 


\section{Conclusions}

In spite that the basic heat conduction equation is very simple, the determination of thermal conductivities from the results of in situ heating tests presents significant challenges. In this study, an analysis of thermal conduction in three argillaceous rocks integrating the results from laboratory measurements and in situ heating tests has been presented. A methodology for the determination of thermal conductivity on the basis of temperature measurements in the rock mass in heating experiments has been proposed. Arguments in favour of this methodology were discussed and it was shown to be more robust than other alternatives. The determined thermal conductivity values in the laboratory and in the field exhibit a very good agreement. In this way, reference thermal conductivity values for the three rocks can be proposed (Table 8). The anisotropic nature of thermal conduction in the three sedimentary rocks should be noted.

An analysis of the impact of potential experimental uncertainties has also been presented. The analysis highlights the high reliability of the determined equivalent thermal conductivity (geometric mean of the thermal conductivity values in the principal directions) as uncertainties on sensor localisation, specific heat and temperature measurements induce only a relatively small dispersion of this parameter. In contrast, the anisotropy ratio was shown to be more sensitive to all types of experimental uncertainties. Uncertainty on the power input exhibits the interesting feature of inducing a clear difference between sensors located in the bedding plane and in the perpendicular direction. That feature allowed the identification of a potential power loss in the ATLAS and the HE-D experiments.

Acknowledgments The support of ANDRA for the development of this work is gratefully acknowledged. Two Technical Meetings for the HE-D experiment and nineteen Technical Meetings for the TER and TED experiments were held and financially supported by ANDRA. Those meetings involved many fruitful discussions, in particular with Yannick Wileveau, Michael Jobmann, Michel Filipi and Nathalie Conil. The important work on the in situ experiments and the laboratory experiments, by ANDRA, EURIDICE, NIRAS.ONDRAF and NAGRA, is duly acknowledged.

\section{References}

ANDRA (2009) Dossier 2009, Référentiel du site Meuse/Haute Marne, Tome 1. C.RP.ADS.A09.0007

Auvray C, Grigc D, Homand F (2005) Essais thermo-mecaniques ouvrage TER1301. ANDRA report C RP 0ENG 05-0378

Bernier F, Li X-L, Bastiaens W (2007) Twenty-five years' geotechnical observation and testing in the Tertiary Boom Clay formation. Géotechnique 57(2):229-237
Bock H (2009) RA experiment: updated review of the rock mechanics properties of the Opalinus Clay of the Mont Terri URL based on laboratory and field testing. Mont Terri Project. Technical report 2008-04

Booker JR, Savvidou C (1985) Consolidation around a point heat source. Int J Numer Anal Methods Geomech 9:173-184

Carslaw HS, Jaeger JC (1946) Conduction of heat in solids. Oxford University Press, Oxford. ISBN: 0198533683

Conil N, Gatimiri B, Armand G (2010) Premiers résultats de l'expérimentation TED. ANDRA report D.RP.AMFS.10.0067

De Bruyn D, Labat S (2002) The second phase of ATLAS: the continuation of a running THM test in the HADES underground research facility at Mol. Eng Geol 64(2002):309-316

Delay J, Rebours H, Vinsot A, Robin P (2007) Scientific investigation in deep wells for nuclear waste disposal studies at the Meuse/ Haute Marne underground research laboratory, Northeastern France. Phys Chem Earth 32:42-57

Djeran I, Bazargan B, Giraud A, Rousset G (1994) Étude expérimentale du comportement Thermo-Hydro-Mécanique de l'argile de Boom. Report ONDRAF 90/10/547

Farouki O (1986) Thermal properties of soils. Trans Tech Pub., Rockport, MA. ISBN: 0878490558, 9780878490554

Fernandez AM (2011) Determination of the specific heat capacity of materials used as confinement barrier at El Cabril. CIEMAT/ DMA/2G208/3/11

Gens A (2011) On the hydromechanical behaviour of argillaceous hard soils-weak rocks. In: XV European conference on soil mechanics \& geotechnical engineering, Athens

Gens A, Vaunat J, Garitte B, Wileveau Y (2007) In situ behaviour of a stiff layered clay subject to thermal loading. Observations and interpretation. Géotechnique 57(2):207-228

Gens A, Sanchez M, Guimaraes L, Do N, Alonso E, Lloret A, Olivella S, Villar MV, Huertas F (2009) A full-scale in situ heating test for highlevel nuclear waste disposal: observations, analysis and interpretation. Géotechnique 59(4):377-399. doi:10.1680/geot.2009.59.4.377

IAEA (2007) Estimation of global inventories of radioactive waste and other radioactive materials. IAEA-TECDOC-1591, Vienna

IAEA (2009) Classification of radioactive waste. International Atomic Energy Agency No. GSG-1, Vienna

Jobmann M, Polster M (2007) Investigation on thermal expansion effects in clay formations. TEE Final Report (DBE-Tec)

Lima Amorim A (2011) Thermo-hydro-mechanical behaviour of two deep Belgian clay formations: Boom and Ypresian Clays. Thesis, Technical University of Catalunya, 253p

Olivella S (1995) Nonisothermal multiphase flow of brine and gas through saline media. Doctoral thesis, Technical University of Catalonia (UPC), Barcelona, Spain

Robertson EC (1988) Thermal properties of rocks. Open-File Report 88-441. US Geological survey

Romero E (1999) Characterization and thermo-hydro-mechanical behaviour of unsaturated Boom Clay: an experimental study. $\mathrm{PhD}$ thesis, Technical University of Catalunya, $405 \mathrm{p}$

Thury M, Bossart P (1999) The Mont Terri rock laboratory, a new international research project in a Mesozoic shale formation, in Switzerland. Eng Geol 52(1999):347-359

Tsang CF, Barnichon JD, Birkholzer J, Li XL, Liu HH, Sillen X (2012) Coupled thermo-hydro-mechanical processes in the near field of a high-level radioactive waste repository in clay formations. Int J Rock Mech Min Sci 49(2012):31-44

Van Cauteren L (1994) Evaluatie van de warmtegeleidbaarheid en de warmtecapaciteit van de Boomse Klei. ONDRAF note nr 94-0605

Wileveau Y (2005) THM behaviour of host rock (HE-D) experiment: progress report. Part 1. Technical Report TR 2005-03. Mont Terri Project 